\title{
Underlay Cognitive Radio Systems with Improper Gaussian Signaling: Outage Performance Analysis
}

\author{
Osama Amin, Senior Member, IEEE, Walid Abediseid, Member, IEEE, and Mohamed-Slim Alouini, Fellow, IEEE
}

\begin{abstract}
Improper Gaussian signaling has the ability over proper (conventional) Gaussian signaling to improve the achievable rate of systems that suffer from interference. In this paper, we study the impact of using improper Gaussian signaling on the performance limits of the underlay cognitive radio system by analyzing the achievable outage probability of both the primary user (PU) and secondary user (SU). We derive the exact outage probability expression of the $\mathrm{SU}$ and construct upper and lower bounds of the PU outage probability which results in formulating an approximate expression of the PU outage probability. This allows us to design the SU signal by adjusting its transmitted power and the circularity coefficient to minimize the $\mathrm{SU}$ outage probability while maintaining a certain PU quality-of-service. Finally, we evaluate the derived expressions for both the SU and the PU and the corresponding adaptive algorithms by numerical results.
\end{abstract}

Index Terms-Cognitive radio, improper Gaussian signaling, asymmetric complex signaling, interference channel, outage probability, average CSI, power allocation, circularity coefficient.

\section{INTRODUCTION}

The innovative progress of wireless technology results in a proliferation of attractive wireless devices and diversity of services. In this era of the massive demand for data throughput and traffic, the shortage of spectrum resources can limit significantly the wireless network performance. Cognitive radio (CR) is a hierarchical dynamic spectrum access technique that can meet the market demand and solve the spectrum scarcity problem. In CR, the secondary user (SU), i.e. unlicensed user, is allowed to access the spectrum as long as it does not affect the transmission quality of the primary user (PU), i.e., licensed user. This can be achieved by either defining transmission periods for the SU as in the interweave technique or by limiting the SU power to avoid unacceptable interference levels at the PU as in the underlay technique [1]. Adopting the underlay technique steers the research focus to mitigate the interference received at the PU end from the SU.

Mitigating the interference in communication systems is a challenging research problem and has received much attention, recently. It is well-known that statistical signal characteristics affect significantly the maximum achievable rate. As oppose to the conventional proper Gaussian signaling, which imposes complex signal with uncorrelated real and imaginary components and equal power for each component, improper

The work of M.-S. Alouini was supported by the Qatar National Research Fund (a member of Qatar Foundation) under NPRP Grant NPRP 5-250-2-087. The statements made herein are solely the responsibility of the authors.

The authors are with Computer, Electrical, and Mathematical Science and Engineering (CEMSE) Division, King Abdullah University of Science and Technology (KAUST) Thuwal, Makkah Province, Saudi Arabia.

E-mail: \{osama.amin, walid.abediseid, slim.alouini\}@kaust.edu.sa.
Gaussian signaling is known to increase the achievable rate over interference channels systems [2], [3].

The term proper signaling was introduced in information theory for the first time by Neeser and Massey in [4], where they defined new second order statistics quantity called pseudo-covariance to fully describe the impropriety of any complex random variable besides the well known conventional covariance. According to their definition, the random variable is said to be proper if the pseudo-covariance vanishes. In [5][7], Picinbono et al. introduced the foundation study of improper complex random vectors and processes. In [8], Schreier and Scharf introduced a comprehensive analysis of the second order statistics of improper random vectors and processes. An overview study of the impropriety role in statistical signal processing modeling and its possible benefits in estimation, detection and time-series analysis was presented by Adali et al. in [9]. A test of the impropriety of complex signals, which is used in different systems such as communication and biomedical systems, was proposed in [10], [11] and a bound measures the degree of impropriety was introduced in [12]. Hence, the following communication signals are classified as improper, pulse amplitude modulation, offset quaternary phase-shift keying, binary phase-shift keying, Gaussian minimum shift keying and the resultant signals from imbalance between the in-phase and quadrature components. To study the impact of improper Gaussian signaling on communication systems, Cadambe et al. investigated the usefulness of this signaling scheme for interference alignment in wireless networks [2]. They were the first who expected the usefulness of this fundamental idea and its possible applications in communication systems. In [13], Taubök studied the influence of improper Gaussian signaling on information theoretic quantities such as entropy, divergence and capacity. Recently, improper Gaussian signaling was applied to communication networks that suffer from interference in order to improve the performance of two user singleinput single-output systems [14], $K$-user multiple-input singleoutput systems [15], $K$-user multiple-input multiple-output systems [3], $Z$-interference channels [16], and multiple-input multiple-output systems with $Z$-interference channel [17].

The achievable rate performance of CR systems using proper Gaussian signaling was studied in [18], [19]. On the other hand, the achievable rate of underlay CR systems that employ improper Gaussian signaling was studied in [20], [21]. In [20], Lameiro et al. assumed that the PU uses proper Gaussian signaling, since there is no control upon it, on the other hand, the SU was designed to use improper Gaussian signaling. Moreover, the SU was assumed to have access to the instantaneous channel state information (CSI) of both the PU and SU communication channels. The instantaneous 
achievable rate of both the PU and SU systems were derived, then the SU power and the circularity coefficient were adjusted to maximize the SU rate while achieving the PU quality-ofservice (QoS). In [21], Gaafar et al. explored the spectrum sharing chances of SU, using improper Gaussian signaling, with full-duplex PU based on the availability of instantaneous CSI of all links at the SU side.

In this paper, we study the outage probability of the underlay CR system employing improper Gaussian signaling at the SU side ${ }^{1}$. We first derive a closed form expression of the SU outage probability. Then, upper and lower bounds, and an approximate expression of the outage probability for the PU are derived. In contrast to [20], [21], where perfect CSI of all links is assumed to be known at the SU nodes, we assume a more practical scenario, where only average CSI is available at the SU nodes. We then adjust the SU power and the circularity coefficient to maximize the SU rate while satisfying the PU QoS.

The rest of the paper is organized as follows. In Section II, we describe the CR system model. Section III studies the outage probability for the PU and SU in the CR system. In Section IV, we adjust the SU signal parameters to maximize the SU rate while satisfying the PU QoS. Then, we validate the performance of the derived bounds and investigate the outage probability performance of SU through simulation examples in Section V. Finally, we conclude the paper in Section VI.

Notation: $|$.$| denotes the absolute value operator and \mathbb{E}$ denotes the statistical expectation.

\section{SYSTEM DESCRIPTION}

\section{A. Preliminaries}

Consider a zero mean scalar random variable $x$ whose conventional variance is defined as $\sigma_{x}^{2}=\mathbb{E}\left[|x|^{2}\right]$, and its pseudo-variance is defined as $\tilde{\sigma}_{x}^{2}=\mathbb{E}\left[x^{2}\right]$ [4].

Definition 1: [4], [23] A complex random variable is called proper if its pseudo-variance is equal to zero, otherwise it is called improper.

Definition 2: [17] The impropriety degree of $x$ is measured by the circularity coefficient that is expressed as

$$
\mathcal{C}_{x}=\frac{\left|\tilde{\sigma}_{x}^{2}\right|}{\sigma_{x}^{2}}
$$

where $0 \leq \mathcal{C}_{x} \leq 1$. If $\mathcal{C}_{x}=0$, we say that the signal is proper, and if $\mathcal{C}_{x}=1$, we have maximally improper signal.

\section{B. Underlay Cognitive Radio System}

In this section, we assume a spectrum sharing system consisting of a SU pair of one transmitter and one receiver that coexist with another licensed communication pair of the PU as depicted in Fig. 1. The communication channels via all links are modeled as Rayleigh fading channels and the noise random variables at the receiver ends are modeled as zero mean white Gaussian additive random variable with variances $\sigma^{2}$. The SU transmitter needs to adjust its power $p_{\mathrm{s}}$ without

\footnotetext{
${ }^{1}$ Part of this paper is accepted for presentation in the ISIT conference [22].
}

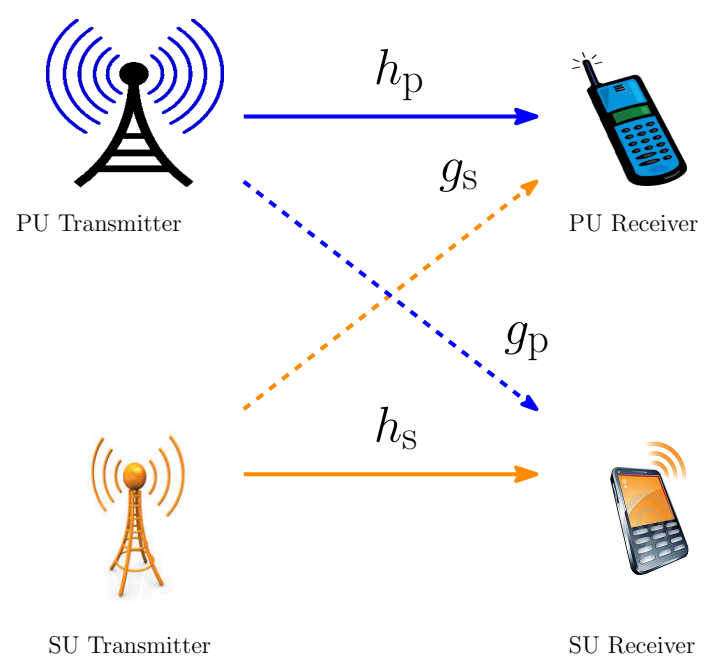

Fig. 1. Underlay CR system model.

affecting the PU QoS. The received signal, denoted as $y_{\mathrm{p}}$, at the PU can be mathematically expressed as

$$
y_{\mathrm{p}}=\sqrt{p_{\mathrm{p}}} h_{\mathrm{p}} x_{\mathrm{p}}+\sqrt{p_{\mathrm{s}}} g_{\mathrm{s}} x_{\mathrm{s}}+n_{\mathrm{p}},
$$

where $p_{\mathrm{p}}$ is the PU transmitted power, $x_{\mathrm{p}}$ is the PU transmitted symbols, which is assumed to be proper Gaussian signal, $x_{\mathrm{s}}$ is the $\mathrm{SU}$ signal with circularity coefficient $\mathcal{C}_{x}, p_{\mathrm{s}}$ is the $\mathrm{SU}$ transmitted power, $h_{\mathrm{p}}$ is the fading channel coefficient of the PU transmission, $g_{\mathrm{s}}$ is the $\mathrm{SU}$ interfering channel coefficient to the PU and $n_{\mathrm{p}}$ is the noise at the PU receiver.

Similarly, the SU receiver suffers from an interference that results from the primary network. Thus, the SU received signal, denoted as $y_{\mathrm{s}}$, may be expressed as,

$$
y_{\mathrm{s}}=\sqrt{p_{\mathrm{s}}} h_{\mathrm{s}} x_{\mathrm{s}}+\sqrt{p_{\mathrm{p}}} g_{\mathrm{p}} x_{\mathrm{p}}+n_{\mathrm{s}},
$$

where $h_{\mathrm{s}}$ is the SU direct link channel coefficient, $g_{\mathrm{p}}$ is the PU interfering channel coefficient to the $\mathrm{SU}$ and $n_{\mathrm{S}}$ is the noise at the SU. Throughout the paper, we assume the interference channel affecting the PU is not strong in order to satisfy its QoS requirement. In addition, we assume the PU interference on the SU is weak so that the SU can operate without the need to increase its power which may violate the PU QoS. Therefore, we deal with the interference signals as noise at both ends.

As a result of employing the improper Gaussian signaling at the SU, the achievable rate of the PU is expressed as [15], [20],

$R_{\mathrm{p}}\left(p_{\mathrm{s}}, \mathcal{C}_{x}\right)=\log _{2}\left(1+\frac{p_{\mathrm{p}}\left|h_{\mathrm{p}}\right|^{2}}{\sigma^{2}+p_{\mathrm{s}}\left|g_{\mathrm{s}}\right|^{2}}\right)+\frac{1}{2} \log _{2}\left(\frac{1-\mathcal{C}_{y_{\mathrm{p}}}^{2}}{1-\mathcal{C}_{I_{\mathrm{p}}}^{2}}\right)$,

where $\mathcal{C}_{y_{\mathrm{p}}}$ and $\mathcal{C}_{I_{\mathrm{p}}}$ are the circularity coefficients of the received and interference-plus-noise signals at the PU, respectively, which are given by

$$
\mathcal{C}_{y_{\mathrm{p}}}=\frac{p_{\mathrm{s}}\left|g_{\mathrm{s}}\right|^{2} \mathcal{C}_{x}}{p_{\mathrm{s}}\left|g_{\mathrm{s}}\right|^{2}+p_{\mathrm{p}}\left|h_{\mathrm{p}}\right|^{2}+\sigma^{2}}, \quad \mathcal{C}_{I_{\mathrm{p}}}=\frac{p_{\mathrm{s}}\left|g_{\mathrm{s}}\right|^{2} \mathcal{C}_{x}}{p_{\mathrm{s}}\left|g_{\mathrm{s}}\right|^{2}+\sigma^{2}} .
$$

From (4) and (5), we observe that the PU rate where the SU employs improper Gaussian signaling is higher than its 
counterpart with proper Gaussian signaling. In the latter, the second term in (4) vanishes, while in the former it gives always a positive value which increases the rate of the PU. After some manipulations, $R_{\mathrm{p}}\left(p_{\mathrm{s}}, \mathcal{C}_{x}\right)$ can be rewritten as

$$
R_{\mathrm{p}}\left(p_{\mathrm{s}}, \mathcal{C}_{x}\right)=\frac{1}{2} \log _{2} \frac{\left(p_{\mathrm{s}}\left|g_{\mathrm{s}}\right|^{2}+p_{\mathrm{p}}\left|h_{\mathrm{p}}\right|^{2}+\sigma^{2}\right)^{2}-p_{\mathrm{s}}^{2}\left|g_{\mathrm{s}}\right|^{4} \mathcal{C}_{x}^{2}}{\left(\sigma^{2}+p_{\mathrm{s}}\left|g_{\mathrm{s}}\right|^{2}\right)^{2}-p_{\mathrm{s}}^{2}\left|g_{\mathrm{s}}\right|^{4} \mathcal{C}_{x}^{2}}
$$

For the SU, the circularity coefficient of the interference term equals zero, thus the SU achievable rate reduces to [15], [20]

$$
\begin{aligned}
R_{\mathrm{s}}\left(p_{\mathrm{s}}, \mathcal{C}_{x}\right) & =\log _{2}\left(1+\frac{p_{\mathrm{s}}\left|h_{\mathrm{s}}\right|^{2}}{p_{\mathrm{p}}\left|g_{\mathrm{p}}\right|^{2}+\sigma^{2}}\right) \\
+ & \frac{1}{2} \log _{2}\left(1-\frac{p_{\mathrm{s}}^{2}\left|h_{\mathrm{s}}\right|^{4} \mathcal{C}_{x}^{2}}{\left(p_{\mathrm{s}}\left|h_{\mathrm{s}}\right|^{2}+p_{\mathrm{p}}\left|g_{\mathrm{p}}\right|^{2}+\sigma^{2}\right)^{2}}\right) .
\end{aligned}
$$

For simplicity, one may rewrite $R_{\mathrm{s}}\left(p_{\mathrm{s}}, \mathcal{C}_{x}\right)$ as

$$
\begin{aligned}
& R_{\mathrm{S}}\left(p_{\mathrm{s}}, \mathcal{C}_{x}\right)= \\
& \quad \frac{1}{2} \log _{2}\left(\frac{p_{\mathrm{s}}^{2}\left|h_{\mathrm{s}}\right|^{4}\left(1-\mathcal{C}_{x}^{2}\right)}{\left(p_{\mathrm{p}}\left|g_{\mathrm{p}}\right|^{2}+\sigma^{2}\right)^{2}}+\frac{2 p_{\mathrm{s}}\left|h_{\mathrm{s}}\right|^{2}}{p_{\mathrm{p}}\left|g_{\mathrm{p}}\right|^{2}+\sigma^{2}}+1\right) .
\end{aligned}
$$

Throughout this paper, we assume the availability of the average CSI of all links at the SU side. Such an assumption is more practical than the perfect knowledge of CSI at the SU side in [20], [21]. The average CSI depends on the path loss and shadowing, which can be measured based on the environment and the distance between the nodes $[24]^{2}$.

\section{Outage Probability Analysis}

The error performance achieved by the optimal coding and decoding strategies is limited by the so-called outage probability. In this section, the overall outage probability of our system is analyzed in detail.

\section{A. Secondary User Outage Probability}

Let $R_{0, s}$ be defined as the target rate of the SU channel. The outage probability of the $\mathrm{SU}, P_{\mathrm{out}, \mathrm{s}}$, is defined as

$$
P_{\text {out }, \mathrm{s}}\left(p_{s}, \mathcal{C}_{x}\right)=\operatorname{Pr}\left[R_{\mathrm{s}}\left(p_{s}, \mathcal{C}_{x}\right)<R_{0, \mathrm{~s}}\right] .
$$

Substituting (8) in (9), we get

$P_{\text {out }, \mathrm{s}}\left(p_{s}, \mathcal{C}_{x}\right)=\operatorname{Pr}\left[\frac{p_{\mathrm{s}}^{2}\left(1-\mathcal{C}_{x}^{2}\right) \gamma_{\mathrm{s}}^{2}}{\left(1+p_{\mathrm{p}} \mathcal{I}_{\mathrm{p}}\right)^{2}}+\frac{2 p_{\mathrm{s}} \gamma_{\mathrm{s}}}{1+p_{\mathrm{p}} \mathcal{I}_{\mathrm{p}}}-\Gamma_{\mathrm{s}}<0\right]$,

where $\Gamma_{\mathrm{s}}=2^{2 R_{0, \mathrm{~s}}}-1, \gamma_{\mathrm{s}}=\left|h_{\mathrm{s}}\right|^{2} / \sigma^{2}$ is an exponential random variable that represents the direct-channel-to-noiseratio of the SU with mean $\mathbb{E}\left\{\gamma_{\mathrm{s}}\right\}=\bar{\gamma}_{\mathrm{s}}$, and $\mathcal{I}_{\mathrm{p}}=\left|g_{\mathrm{p}}\right|^{2} / \sigma^{2}$ is

\footnotetext{
${ }^{2}$ The distances between the SU and the PU nodes can be estimated by using the received signal from the PU nodes, or knowing the location of the licensed base station or access points which is fixed and known for all nodes. The distance between the direct link PU transmitter and receiver can be computed using the law of cosines, where the distance between the SU transmitter and the PU receiver is estimated during the PU uplink transmission. Alternatively, one can know the distance through exchanging some information between the $\mathrm{PU}$ and the SU for monitoring purposes [25].
}

an exponential random variable with mean $\mathbb{E}\left\{\mathcal{I}_{\mathrm{p}}\right\}=\overline{\mathcal{I}}_{\mathrm{p}}$ that represents the interference-channel-to-noise-ratio of the PU on the SU. By solving the inequality inside the probability of (10), one can show that the conditional SU outage probability (conditioned on $\mathcal{I}_{\mathrm{p}}$ ) is given by

$$
\begin{aligned}
P_{\text {out }, \mathrm{s}}\left(p_{s}, \mathcal{C}_{x} \mid \mathcal{I}_{\mathrm{p}}\right) & =\int_{0}^{\gamma_{\mathrm{s}_{\mathrm{o}}}} \frac{1}{\overline{\bar{\gamma}}_{\mathrm{s}}} \exp \left(-\frac{u}{\bar{\gamma}_{\mathrm{s}}}\right) d u \\
& =1-\exp \left(-\frac{p_{\mathrm{p}} \mathcal{I}_{\mathrm{p}}+1}{1-\mathcal{C}_{x}^{2}} \Psi_{\mathrm{s}}\left(\mathcal{C}_{x}\right)\right),
\end{aligned}
$$

where $\gamma_{\mathrm{s}_{\mathrm{o}}}$ represents the non-negative root that satisfies the inequality in (10) and is expressed as

$$
\gamma_{\mathrm{s}_{\mathrm{o}}}=\frac{\bar{\gamma}_{\mathrm{s}}\left(p_{\mathrm{p}} \mathcal{I}_{\mathrm{p}}+1\right) \Psi_{\mathrm{s}}\left(\mathcal{C}_{x}\right)}{\left(1-\mathcal{C}_{x}^{2}\right)}
$$

where $\Psi_{\mathrm{s}}\left(\mathcal{C}_{x}\right)=\left(\sqrt{1+\Gamma_{\mathrm{s}}\left(1-\mathcal{C}_{x}^{2}\right)}-1\right) /\left(p_{\mathrm{s}} \bar{\gamma}_{\mathrm{s}}\right)$. By averaging (11) over the exponential statistics of $\mathcal{I}_{\mathrm{p}}$, we obtain

$$
\begin{aligned}
& P_{\text {out }, \mathrm{s}}\left(p_{s}, \mathcal{C}_{x}\right)=\mathbb{E}_{\mathcal{I}_{\mathrm{p}}}\left\{P_{\text {out }, \mathrm{s}}\left(p_{\mathrm{s}}, \mathcal{C} \mid \mathcal{I}_{\mathrm{p}}\right)\right\} \\
& \quad=1-\frac{1-\mathcal{C}_{x}^{2}}{1-\mathcal{C}_{x}^{2}+\overline{\mathcal{I}}_{\mathrm{p}} p_{\mathrm{p}} \Psi_{\mathrm{s}}\left(\mathcal{C}_{x}\right)} \exp \left(-\frac{\Psi_{\mathrm{s}}\left(\mathcal{C}_{x}\right)}{1-\mathcal{C}_{x}^{2}}\right) .
\end{aligned}
$$

For $\mathcal{C}_{x}=0$, the above outage probability reduces to the proper Gaussian signaling scheme that is expressed as

$$
P_{\text {out }, \mathrm{s}}\left(p_{s}, 0\right)=1-\frac{\exp \left(-\Psi_{\mathrm{s}}(0)\right)}{1+\overline{\mathcal{I}}_{\mathrm{p}} p_{\mathrm{p}} \Psi_{\mathrm{s}}(0)} .
$$

On the other extreme, the maximally improper signaling scheme, i.e., when $\mathcal{C}_{x} \rightarrow 1$, yields

$$
P_{\text {out }, \mathrm{s}}\left(p_{s}, 1\right)=\lim _{\mathcal{C}_{x} \rightarrow 1} P_{\text {out }, \mathrm{s}}\left(p_{s}, \mathcal{C}_{x}\right)=1-\frac{\exp \left(-\frac{\Gamma_{\mathrm{s}}}{2 p_{\mathrm{s}} \bar{\gamma}_{s}}\right)}{1+\frac{p_{\mathrm{p}} \overline{\mathcal{I}}_{\mathrm{p}} \Gamma_{\mathrm{s}}}{2 p_{\mathrm{s}} \bar{\gamma}_{s}}}
$$

\section{B. Primary User Outage Probability}

Similar to the above subsection, our goal here is to find a closed form for the PU outage probability in terms of the signal and channels parameters. Let $R_{0, p}$ be defined as the target rate of the SU channel. The outage probability of the $\mathrm{PU}, P_{\text {out }, \mathrm{p}}\left(p_{s}, \mathcal{C}_{x}\right)$, is defined as

$$
P_{\text {out }, \mathrm{p}}\left(p_{s}, \mathcal{C}_{x}\right)=\operatorname{Pr}\left[R_{\mathrm{p}}\left(p_{s}, \mathcal{C}_{x}\right)<R_{0, \mathrm{p}}\right] .
$$

Substituting (6) in (16), we get

$$
\begin{aligned}
P_{\text {out }, \mathrm{p}}\left(p_{s}, \mathcal{C}_{x}\right)= & \operatorname{Pr}\left[\gamma_{\mathrm{p}}^{2}+\frac{2}{p_{\mathrm{p}}}\left(p_{\mathrm{s}} \mathcal{I}_{\mathrm{s}}+1\right) \gamma_{\mathrm{p}}\right. \\
& \left.-\frac{\Gamma_{\mathrm{p}}}{p_{\mathrm{p}}^{2}}\left[\left(p_{\mathrm{s}} \mathcal{I}_{\mathrm{s}}+1\right)^{2}-p_{\mathrm{s}}^{2} \mathcal{I}_{\mathrm{s}}^{2} \mathcal{C}_{x}^{2}\right]<0\right],
\end{aligned}
$$

where $\Gamma_{\mathrm{p}}=2^{2 R_{0, \mathrm{p}}}-1, \gamma_{\mathrm{p}}=\left|h_{\mathrm{p}}\right|^{2} / \sigma^{2}$ is an exponential random variable that represents the direct-channel-to-noiseratio of the PU with mean $\mathbb{E}\left\{\gamma_{\mathrm{p}}\right\}=\bar{\gamma}_{\mathrm{p}}$ and $\mathcal{I}_{\mathrm{s}}=\left|g_{\mathrm{s}}\right|^{2} / \sigma^{2}$ is an exponential random variable with mean $\mathbb{E}\left\{\mathcal{I}_{\mathrm{s}}\right\}=\overline{\mathcal{I}}_{\mathrm{s}}$ that represents the interference-channel-to-noise-ratio of the SU on the PU. By solving the inequality that appears inside the probability of (17), one can show that the conditional PU outage probability (conditioned on $\mathcal{I}_{\mathrm{s}}$ ) is given by

$$
P_{\text {out }, \mathrm{p}}\left(p_{s}, \mathcal{C}_{x} \mid \mathcal{I}_{\mathrm{s}}\right)=1-\exp \left(-\gamma_{\mathrm{p}_{o}} / \bar{\gamma}_{\mathrm{p}}\right),
$$


where $\gamma_{p_{\circ}}$ represents the root that satisfies the inequality in (17) and is found to be

$$
\gamma_{\mathrm{p}_{\mathrm{o}}}=\gamma_{\mathrm{p}}\left(p_{\mathrm{s}} \mathcal{I}_{\mathrm{s}}+1\right) \Psi_{\mathrm{p}}\left(\frac{p_{\mathrm{s}} \mathcal{I}_{\mathrm{s}} C_{x}}{p_{\mathrm{s}} \mathcal{I}_{\mathrm{s}}+1}\right)
$$

and $\Psi_{\mathrm{p}}(x)=\frac{\sqrt{1+\Gamma_{\mathrm{p}}\left[1-x^{2}\right]}-1}{p_{\mathrm{p}} \bar{\gamma}_{\mathrm{p}}}$.

Averaging (18) over the statistics of $\mathcal{I}_{\mathrm{s}}$, we get

$$
\begin{aligned}
& P_{\text {out }, \mathrm{p}}\left(p_{s}, \mathcal{C}_{x}\right)=\mathbb{E}_{\mathcal{I}_{\mathrm{s}}}\left\{P_{\text {out }, \mathrm{p}}\left(p_{s}, \mathcal{C}_{x} \mid \mathcal{I}_{\mathrm{s}}\right)\right\} \\
& \quad=1-\int_{0}^{\infty} \frac{1}{\overline{\mathcal{I}}_{\mathrm{s}}} \exp \left(-\frac{u}{\overline{\mathcal{I}}_{\mathrm{s}}}-\left(p_{\mathrm{s}} u+1\right) \Psi_{\mathrm{p}}\left(\frac{\mathcal{C}_{x} p_{\mathrm{s}} u}{1+p_{\mathrm{s}} u}\right)\right) d u .
\end{aligned}
$$

After changing the variables, $z=p_{\mathrm{s}} u$ in (20), we obtain the reformulated PU outage probability integral as

$$
\begin{aligned}
& P_{\text {out }, \mathrm{p}}\left(p_{s}, \mathcal{C}_{x}\right)=1- \\
& \frac{1}{p_{\mathrm{s}} \overline{\mathcal{I}}_{\mathrm{s}}} \int_{0}^{\infty} \exp \left(-\frac{z}{p_{\mathrm{s}} \overline{\mathcal{I}}_{\mathrm{s}}}-(z+1) \Psi_{\mathrm{p}}\left(\frac{\mathcal{C}_{x} z}{1+z}\right)\right) d z .
\end{aligned}
$$

Unfortunately, it is very difficult to obtain a closed form expression for the aforementioned PU integral for any value of $\mathcal{C}_{x}$, except for the case when $\mathcal{C}_{x}=0$, i.e., for proper Gaussian signaling. In this scenario, the PU outage probability simplifies to

$$
P_{\text {out }, \mathrm{p}}\left(p_{\mathrm{s}}, 0\right)=1-\frac{\exp \left(-\Psi_{\mathrm{p}}(0)\right)}{1+\overline{\mathcal{I}}_{\mathrm{s}} p_{\mathrm{s}} \Psi_{\mathrm{p}}(0)} .
$$

For values of $0<\mathcal{C}_{x} \leq 1$, i.e., for improper Gaussian signaling schemes, we will resort to deriving lower and upper bounds of the PU outage probability to study the PU outage probability behavior limits.

1) Lower Bound of the PU Outage Probability: First, we simplify the PU outage probability integral in (21) as

$$
\begin{gathered}
P_{\text {out }, \mathrm{p}}\left(p_{\mathrm{s}}, \mathcal{C}_{x}\right)=1-\frac{1}{p_{\mathrm{s}} \overline{\mathcal{I}}_{\mathrm{s}}} \int_{0}^{\infty} \exp \left(-\frac{z}{p_{\mathrm{s}} \overline{\mathcal{I}}_{\mathrm{s}}}+\frac{z+1}{p_{\mathrm{p}} \bar{\gamma}_{\mathrm{p}}}\right) \times \\
\exp \left(-\frac{\sqrt{1+\Gamma_{\mathrm{p}}}}{p_{\mathrm{p}} \bar{\gamma}_{\mathrm{p}}} \sqrt{\left.\left(1-\frac{\Gamma_{\mathrm{p}} \mathcal{C}_{x}^{2}}{1+\Gamma_{\mathrm{p}}}\right) z^{2}+2 z+1\right)} d z .\right.
\end{gathered}
$$

One way to lower bound the PU outage probability is by using the fact that for $z \geq 0$, we have

$$
\left(1-\frac{\Gamma_{\mathrm{p}} \mathcal{C}_{x}^{2}}{1+\Gamma_{\mathrm{p}}}\right) z^{2}+2 z+1 \geq\left(\sqrt{1-\frac{\Gamma_{\mathrm{p}} \mathcal{C}_{x}^{2}}{1+\Gamma_{\mathrm{p}}}} z+1\right)^{2}
$$

In this case, we may lower bound $P_{\text {out }, \mathrm{p}}\left(p_{\mathrm{s}}, \mathcal{C}_{x}\right)$ in (23) for any value of $\mathcal{C}_{x}$ as

$$
\begin{gathered}
P_{\text {out }, \mathrm{p}}\left(p_{\mathrm{s}}, \mathcal{C}_{x}\right) \geq 1-\frac{1}{p_{\mathrm{s}} \overline{\mathcal{I}}_{\mathrm{s}}} \exp \left(-\frac{1}{p_{\mathrm{p}} \bar{\gamma}_{\mathrm{p}}}\left(\sqrt{1+\Gamma_{\mathrm{p}}}-1\right)\right) \times \\
\int_{0}^{\infty} \exp \left(-\frac{z}{p_{\mathrm{s}} \overline{\mathcal{I}}_{\mathrm{s}}}-\frac{z}{p_{\mathrm{p}} \bar{\gamma}_{\mathrm{p}}}\left(\sqrt{1+\Gamma_{\mathrm{p}}\left(1-\mathcal{C}_{x}^{2}\right)}-1\right)\right) d z \\
=1-\frac{\exp \left(-\Psi_{\mathrm{p}}(0)\right)}{1+p_{\mathrm{s}} \overline{\mathcal{I}}_{\mathrm{s}} \Psi_{\mathrm{p}}\left(\mathcal{C}_{x}\right)} \triangleq P_{\text {out }, \mathrm{p}}^{\mathrm{LB}}\left(p_{\mathrm{s}}, \mathcal{C}_{x}\right) .
\end{gathered}
$$

Note that the lower bound in (25) reduces to the PU outage probability exact expression in (22) at $\mathcal{C}_{x}=0$.
2) Upper Bound of the PU Outage Probability: One can derive an upper bound for $z \geq 0$, by using the fact that $1>\left(1-\Gamma_{\mathrm{p}} \mathcal{C}_{x}^{2} /\left(1+\Gamma_{\mathrm{p}}\right)\right)$, for all $\mathcal{C}_{x}>0$. However, the drawback of using such bound is that the dependency of the outage expression on the impropriety parameter $\mathcal{C}_{x}$ vanishes and results in a loose PU outage probability upper bound. To obtain a tighter bound, we split the integral in (23) as follows

$$
\begin{aligned}
& P_{\text {out }, \mathrm{p}}\left(p_{\mathrm{s}}, \mathcal{C}_{x}\right)=1 \\
& -\frac{1}{p_{\mathrm{s}} \overline{\mathcal{I}}_{\mathrm{s}}} \int_{0}^{\alpha} \exp \left(-\frac{z}{p_{\mathrm{s}} \overline{\mathcal{I}}_{\mathrm{s}}}-(z+1) \Psi_{\mathrm{p}}\left(\frac{\mathcal{C}_{x} z}{1+z}\right)\right) d z \\
& \quad-\frac{1}{p_{\mathrm{s}} \overline{\mathcal{I}}_{\mathrm{s}}} \int_{\alpha}^{\infty} \exp \left(-\frac{z}{p_{\mathrm{s}} \overline{\mathcal{I}}_{\mathrm{s}}}-(z+1) \Psi_{\mathrm{p}}\left(\frac{\mathcal{C}_{x} z}{1+z}\right)\right) d z \\
& \stackrel{(a)}{\leq} 1-\frac{1}{p_{\mathrm{s}} \overline{\mathcal{I}}_{\mathrm{s}}} \int_{0}^{\alpha} \exp \left(-\frac{z}{p_{\mathrm{s}} \overline{\mathcal{I}}_{\mathrm{s}}}-(z+1) \Psi_{\mathrm{p}}(0)\right) d z \\
& \quad-\frac{1}{p_{\mathrm{s}} \overline{\mathcal{I}}_{\mathrm{s}}} \int_{\alpha}^{\infty} \exp \left(-\frac{z}{p_{\mathrm{s}} \overline{\mathcal{I}}_{\mathrm{s}}}-(z+1) \Psi_{\mathrm{p}}\left(\frac{\mathcal{C}_{x} \alpha}{1+\alpha}\right)\right) d z \\
& \triangleq P_{\text {out }, \mathrm{p}}^{\mathrm{UB}}\left(p_{\mathrm{s}}, \mathcal{C}_{x}, \alpha\right),
\end{aligned}
$$

where $(a)$ follows from using the fact that for $z \geq 0$ we have $\left(\frac{p_{\mathrm{s}} z}{1+p_{\mathrm{s}} z}\right) \geq 0$ in the first integral, and the fact that for $z \geq \alpha$, we have $\left(\frac{p_{\mathrm{s}} z}{1+p_{\mathrm{s}} z}\right) \geq\left(\frac{p_{\mathrm{s}} \alpha}{1+p_{\mathrm{s}} \alpha}\right)$ in the second integral. By evaluating the integrals, we get

$$
\begin{aligned}
& P_{\text {out }, \mathrm{p}}^{\mathrm{UB}}\left(p_{\mathrm{s}}, \mathcal{C}_{x}, \alpha\right)=1+\frac{\exp \left(-\frac{\alpha}{\overline{\mathcal{I}}_{\mathrm{s}} p_{\mathrm{s}}}-(\alpha+1) \Psi_{\mathrm{p}}(0)\right)}{1+p_{\mathrm{s}} \overline{\mathcal{I}}_{\mathrm{s}} \Psi_{\mathrm{p}}(0)} \\
& -\frac{\exp \left(\Psi_{\mathrm{p}}(0)\right)}{1+p_{\mathrm{s}} \overline{\mathcal{I}}_{\mathrm{s}} \Psi_{\mathrm{p}}(0)}-\frac{\exp \left(-\frac{\alpha}{\overline{\mathcal{I}}_{\mathrm{s}} p_{\mathrm{s}}}-(\alpha+1) \Psi_{\mathrm{p}}\left(\frac{\mathcal{C}_{x} \alpha}{1+\alpha}\right)\right)}{1+p_{\mathrm{s}} \overline{\mathcal{I}}_{\mathrm{s}} \Psi_{\mathrm{p}}\left(\frac{\mathcal{C}_{x} \alpha}{1+\alpha}\right)} .
\end{aligned}
$$

From (27), we observe that the upper bound reduces to the outage probability of the PU under proper Gaussian signaling assumption (22), i.e., $P_{\text {out }, \mathrm{p}}^{\mathrm{UB}}\left(p_{\mathrm{s}}, 0, \alpha\right)=P_{\text {out }, \mathrm{p}}\left(p_{\mathrm{s}}, 0\right)$. The best upper bound can be obtained by finding the value of $\alpha$ that minimizes $P_{\text {out }, \mathrm{p}}^{\mathrm{UB}}\left(p_{\mathrm{s}}, \mathcal{C}_{x}\right)$, i.e.,

$$
P_{\text {out }, \mathrm{p}}^{\mathrm{UB}}\left(p_{\mathrm{s}}, \mathcal{C}_{x}\right)=\min _{\alpha \geq 0} \quad P_{\text {out }, \mathrm{p}}^{\mathrm{UB}}\left(p_{\mathrm{s}}, \mathcal{C}_{x}, \alpha\right),
$$

which can be found by solving the following equation

$$
\begin{aligned}
& \exp \left((\alpha+1)\left[\Psi_{\mathrm{p}}\left(\frac{\mathcal{C}_{x} \alpha}{\alpha+1}\right)-\Psi_{\mathrm{p}}(0)\right]\right) \\
& +\left(1+\frac{\frac{p_{\mathrm{S}} \overline{\mathcal{I}}_{\mathrm{s}}}{1+\alpha}}{1+p_{\mathrm{s}} \overline{\mathcal{I}}_{\mathrm{s}} \Psi_{\mathrm{p}}\left(\frac{\mathcal{C}_{x} \alpha}{\alpha+1}\right)}\right)\left(\frac{\frac{\mathcal{C}_{x}^{2} \alpha}{(1+\alpha)^{2}}}{1+p_{\mathrm{p}} \bar{\gamma}_{\mathrm{p}} \Psi_{\mathrm{p}}\left(\frac{\mathcal{C}_{x} \alpha}{\alpha+1}\right)}\right) \\
& \times \frac{\frac{p_{\mathrm{s}} \overline{\mathcal{I}}_{\mathrm{s}}}{p_{\mathrm{p}} \bar{\gamma}_{\mathrm{p}}}}{1+p_{\mathrm{p}}}-1=0 .
\end{aligned}
$$

3) Approximate Expression of the PU Outage Probability: To find a more tractable expression than (27), we consider the 
following approximate outage probability expression, denoted by $\tilde{P}_{\text {out,p }}\left(p_{\mathrm{s}}, \mathcal{C}_{x}, \alpha\right)$, which coincides with (27) at $\alpha \gg 1$,

$$
\begin{gathered}
\tilde{P}_{\text {out }, \mathrm{p}}\left(p_{\mathrm{s}}, \mathcal{C}_{x}, \alpha\right)=1+\frac{\exp \left(-\frac{\alpha}{\overline{\mathcal{I}}_{\mathrm{s}} p_{\mathrm{s}}}-\alpha \Psi_{\mathrm{p}}(0)\right)}{1+p_{\mathrm{s}} \overline{\mathcal{I}}_{\mathrm{s}} \Psi_{\mathrm{p}}(0)} \\
-\frac{\exp \left(-\Psi_{\mathrm{p}}(0)\right)}{1+p_{\mathrm{s}} \overline{\mathcal{I}}_{\mathrm{s}} \Psi_{\mathrm{p}}(0)}-\frac{\exp \left(-\frac{\alpha}{\overline{\mathcal{I}}_{\mathrm{s}} p_{\mathrm{s}}}-\alpha \Psi_{\mathrm{p}}\left(\mathcal{C}_{x}\right)\right)}{1+p_{\mathrm{s}} \overline{\mathcal{I}}_{\mathrm{s}} \Psi_{\mathrm{p}}\left(\mathcal{C}_{x}\right)} .
\end{gathered}
$$

This approximate outage probability expression reduces to the exact expression at $\mathcal{C}_{x}=0$, i.e., $\tilde{P}_{\text {out }, \mathrm{p}}\left(p_{\mathrm{s}}, 0, \alpha\right)=$ $P_{\text {out }, \mathrm{p}}\left(p_{\mathrm{s}}, 0\right)$. To study the behavior of $\tilde{P}_{\text {out }, \mathrm{p}}\left(p_{\mathrm{s}}, \mathcal{C}_{x}, \alpha\right)$ for all values of $\alpha \geq 0$, we first consider the derivation of $\tilde{P}_{\text {out }, \mathrm{p}}\left(p_{\mathrm{s}}, \mathcal{C}_{x}, \alpha\right)$, which is found to be

$$
\begin{aligned}
\frac{\partial \tilde{P}_{\text {out }, \mathrm{p}}\left(p_{\mathrm{s}}, \mathcal{C}_{x}, \alpha\right)}{\partial \alpha}= & \overline{\mathcal{I}}_{\mathrm{s}} p_{\mathrm{s}} \exp \left(-\frac{\alpha}{\overline{\mathcal{I}}_{\mathrm{s}} p_{\mathrm{s}}}-\alpha \Psi_{\mathrm{p}}\left(\mathcal{C}_{x}\right)\right) \\
& -\overline{\mathcal{I}}_{\mathrm{s}} p_{\mathrm{s}} \exp \left(-\frac{\alpha}{\overline{\mathcal{I}}_{\mathrm{s}} p_{\mathrm{s}}}-\alpha \Psi_{\mathrm{p}}(0)\right) .
\end{aligned}
$$

Since $\Psi_{\mathrm{p}}(0) \geq \Psi_{\mathrm{p}}\left(\mathcal{C}_{x}\right)$, then $\frac{\partial \tilde{P}_{\text {out }, \mathrm{p}}\left(p_{\mathrm{s}}, \mathcal{C}_{x}, \alpha\right)}{\partial \alpha} \geq 0$. This observation proves that $\tilde{P}_{\text {out }, \mathrm{p}}\left(p_{\mathrm{s}}, \mathcal{C}_{x}, \alpha\right)$ is monotonically increasing in $\alpha$. As a result, it is interesting to compare the behavior of $\tilde{P}_{\text {out, } \mathrm{p}}\left(p_{\mathrm{s}}, \mathcal{C}_{x}, \alpha\right)$ with the upper bound, the lower bound and the exact PU outage probability expressions. As for the lower bound comparison, we express $\tilde{P}_{\text {out }, \mathrm{p}}\left(p_{\mathrm{s}}, \mathcal{C}_{x}, 0\right)$ in terms of the lower bound (25) as follows

$$
\begin{aligned}
\tilde{P}_{\text {out }, \mathrm{p}}\left(p_{\mathrm{s}}, \mathcal{C}_{x}, 0\right)= & 1+\frac{1}{1+p_{\mathrm{s}} \overline{\mathcal{I}}_{\mathrm{s}} \Psi_{\mathrm{p}}\left(\mathcal{C}_{x}\right)}-\frac{1-\exp \left(-\Psi_{\mathrm{p}}(0)\right)}{1+p_{\mathrm{s}} \overline{\mathcal{I}}_{\mathrm{s}} \Psi_{\mathrm{p}}(0)} \\
= & P_{\text {out }, \mathrm{p}}^{\mathrm{LB}}\left(p_{\mathrm{s}}, \mathcal{C}_{x}\right)-\left[1-\exp \left(-\Psi_{\mathrm{p}}(0)\right)\right] \times \\
& {\left[\frac{1}{1+p_{\mathrm{s}} \overline{\mathcal{I}}_{\mathrm{s}} \Psi_{\mathrm{p}}\left(\mathcal{C}_{x}\right)}-\frac{1}{1+p_{\mathrm{s}} \overline{\mathcal{I}}_{\mathrm{s}} \Psi_{\mathrm{p}}(0)}\right] } \\
\leq & P_{\text {out }, \mathrm{p}}^{\mathrm{LB}}\left(p_{\mathrm{s}}, \mathcal{C}_{x}\right) .
\end{aligned}
$$

Then, one can prove that for all values of $\alpha \geq 0$, the approximate PU outage probability expression is upper-bounded by $P_{\text {out }, \mathrm{p}}^{\mathrm{UB}}\left(p_{\mathrm{s}}, \mathcal{C}_{x}, \alpha\right)$ (see Appendix A). Now, we summarize the interesting characteristics of $\tilde{P}_{\text {out, } \mathrm{p}}$ as follows:

- $\tilde{P}_{\text {out }, \mathrm{p}}\left(p_{\mathrm{s}}, \mathcal{C}_{x}, \alpha\right)$ is monotonically increasing in $\alpha$

- $\tilde{P}_{\text {out }, \mathrm{p}}\left(p_{\mathrm{s}}, \mathcal{C}_{x}, 0\right) \leq P_{\text {out }, \mathrm{p}}^{\mathrm{LB}}\left(p_{\mathrm{s}}, \mathcal{C}_{x}\right)$

- $\tilde{P}_{\text {out }, \mathrm{p}}\left(p_{\mathrm{s}}, \mathcal{C}_{x}, \alpha\right) \leq P_{\text {out }, \mathrm{p}}^{\mathrm{UB}}\left(p_{\mathrm{s}}, \mathcal{C}_{x}\right)$

- $\tilde{P}_{\text {out }, \mathrm{p}}\left(p_{\mathrm{s}}, \mathcal{C}_{x}, \alpha\right) \rightarrow P_{\text {out }, \mathrm{p}}^{\mathrm{UB}}\left(p_{\mathrm{s}}, \mathcal{C}_{x}\right)$ as $\alpha \rightarrow \infty$

Hence, one can prove that there exists a unique value of $\alpha$, say $\alpha^{*}$ that makes $\tilde{P}_{\text {out }, \mathrm{p}}\left(p_{\mathrm{s}}, \mathcal{C}_{x}, \alpha\right)$ equivalent to the exact PU outage probability, i.e., $\tilde{P}_{\text {out }, \mathrm{p}}\left(p_{\mathrm{s}}, \mathcal{C}_{x}, \alpha^{*}\right)=P_{\text {out }, \mathrm{p}}\left(p_{\mathrm{s}}, \mathcal{C}_{x}\right)$. To further clarify this point, we plot the lower bound, upper bound, approximate and exact expression versus $\alpha$ in Fig. 2. A good estimate of $P_{\text {out }, \mathrm{p}}\left(p_{\mathrm{s}}, \mathcal{C}_{x}\right)$ can then be evaluated using $\tilde{P}_{\text {out }, \mathrm{p}}\left(p_{\mathrm{s}}, \mathcal{C}_{x}, \alpha\right)$ at $\alpha=\alpha_{\mathrm{UB}}$ where $\alpha_{\mathrm{UB}}$ is computed from (29).

\section{Adaptive Signal Design}

In this section, we propose an approach to improve the SU outage performance by adjusting the SU signal parameters $p_{\mathrm{s}}$ and $\mathcal{C}_{x}$ while maintaining predetermined PU QoS represented by an outage probability threshold for a target rate $R_{0, \mathrm{p}}$. Note that the licensed spectrum is assumed to be assigned

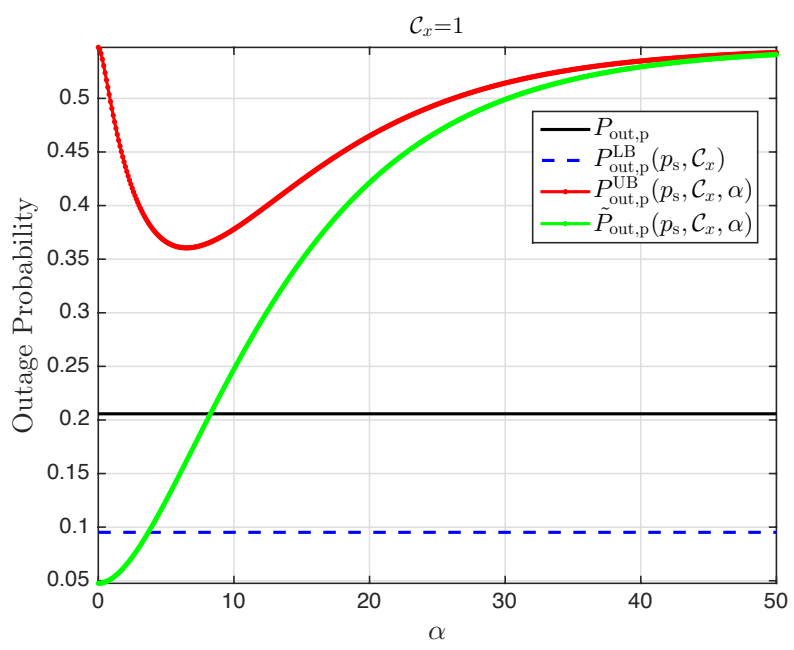

Fig. 2. A comparison between the exact PU outage probability, lower bound, upper bound and approximate expressions versus $\alpha$.

only to the authorized users, i.e. PUs. However due to other factors such as frequency reuse in cellular networks, there might be a received aggregate interference from other cells. In this scenarios, the PU may protect itself against possible interference sources and adjust the transmitted power to achieve the required transmission quality. From the PU point of view, the system is designed to achieve a maximum outage probability, say $P_{\text {out,th }}$, considering an acceptable interference margin power, say $\mathcal{P}_{\text {int }}$ [26]. The design value of $\mathcal{P}_{\text {int }}$ is computed according to the communication network characteristic for a target PU link budget. In this case, $P_{\text {out,th }}$ is expressed in terms of $\mathcal{P}_{\text {int }}$ (assuming the interference sources are all symmetric Gaussian distributed to reflect the worst case scenario) as

$$
\begin{aligned}
P_{\text {out }, \text { th }} & =\operatorname{Pr}\left[\log _{2}\left(1+\frac{p_{\mathrm{p}}\left|h_{\mathrm{p}}\right|^{2}}{\sigma^{2}+\mathcal{P}_{\text {int }}}\right)<R_{0, \mathrm{p}}\right] \\
& =1-\exp \left(-\frac{1+\mathcal{I}_{\max }}{p_{\mathrm{p}} \bar{\gamma}_{\mathrm{p}}}\left(\sqrt{1+\Gamma_{\mathrm{p}}}-1\right)\right),
\end{aligned}
$$

where $\mathcal{I}_{\max } \triangleq \mathcal{P}_{\text {int }} / \sigma^{2}$ is defined as the maximum allowable interference-to-noise ratio at the PU receiver end.

Although the PU is unaware of any other users that use its spectrum locally, i.e., in its coverage area, it may be subjected to aggregate interference sources as occurs in cellular networks. As a result, primary transmitters tend to consider suitable interference power margin to keep a specific QoS as discussed in [26]. As such, the PU must adjust its power according to the following relation,

$$
p_{\mathrm{p}}=\left(\frac{1+\mathcal{I}_{\max }}{\bar{\gamma}_{\mathrm{p}} \log \left(1-P_{\mathrm{out}, \mathrm{th}}\right)}\right)\left(1-\sqrt{1+\Gamma_{\mathrm{p}}}\right) .
$$

It is important to note that the SU has to consider the PU design conditions while adjusting its signal parameters. For our design purpose, we consider first the proper Gaussian signaling scheme as a benchmark, and then we propose three different designs based on the PU lower-bound, upper-bound 
and approximate outage probability expressions. This will be introduced in the sequel.

\section{A. Proper Gaussian signaling Design}

For the proper signaling scheme, the SU system adjusts its transmission power, $p_{\mathrm{s}}$, to suppress its interference on the $\mathrm{PU}$ in order to maintain its required QoS level. Specifically, $p_{\mathrm{s}}$ needs to be computed such that $P_{\text {out }, \mathrm{p}}\left(p_{\mathrm{s}}, 0\right)$ satisfies a predefined outage threshold $P_{\text {out,th }}$ for a given target rate, $R_{0, \mathrm{p}}$, i.e.,

$$
P_{\text {out }, \mathrm{p}}\left(p_{\mathrm{s}}, 0\right)=P_{\text {out,th }} \text {. }
$$

After some simplifications, the value of $p_{\mathrm{s}}$ that satisfies (35) can be shown to be expressed as

$$
p_{\mathrm{s}}=\frac{\exp \left(-\Psi_{\mathrm{p}}(0)\right)-\left(1-P_{\text {out }, \mathrm{th}}\right)}{\Psi_{\mathrm{p}}(0)\left(1-P_{\text {out }, \mathrm{th}}\right) \overline{\mathcal{I}}_{\mathrm{s}}} .
$$

From (36), one can conclude that the SU may access the spectrum, while maintaining the PU QoS requirements, if the following condition is satisfied

$$
\exp \left(-\Psi_{\mathrm{p}}(0)\right)>\left(1-P_{\text {out }, \text { th }}\right),
$$

otherwise, the SU must remain silent. The condition in (37) is valid as long as the maximum marginal interference-to-noise ratio is strictly greater than zero, i.e., $\mathcal{I}_{\max }>0$ (see(33)). Moreover, it indicates that the PU is not fully loaded ${ }^{3}$. Since $\mathcal{I}_{\max }$ represents the maximum interference temperature at the PU side, then limiting $\mathcal{I}_{\max }$ is equivalent to maintaining the PU outage probability at $P_{\text {out,th }}$ [27]. However, this one-toone relation is only valid for proper Gaussian interference source(s). In the remaining of this paper, we do not design the cognitive radio system based on $\mathcal{I}_{\max }$ because this one-toone relation with $P_{\text {out,th }}$ is not valid for improper Gaussian interference source(s). Therefore, we adopt the outage probability for a target rate to reflect the PU QoS constraint.

\section{B. Improper Gaussian signaling Design}

For the improper Gaussian signaling scheme, we have an additional design parameter, $\mathcal{C}_{x}$, which controls the signal impropriety. We expect to have an infinite $\left(p_{\mathrm{s}}, \mathcal{C}_{x}\right)$ pairs that satisfy the PU QoS. This design flexibility gives us the opportunity to target another objective to optimize while maintaining the PU performance requirements.

In this work, the $\mathrm{SU}$ signal parameters $p_{\mathrm{s}}$ and $\mathcal{C}_{x}$ are adaptably computed to minimize the $\mathrm{SU}$ outage probability while satisfying the PU QoS, i.e., $P_{\text {out,p }}\left(p_{\mathrm{s}}, \mathcal{C}_{x}\right) \leq P_{\text {out,th}}$. For this purpose, we formulate the following optimization problem,

$$
\begin{aligned}
\min _{p_{\mathrm{s}}, \mathcal{C}_{x}} & P_{\text {out }, \mathrm{s}}\left(p_{\mathrm{s}}, \mathcal{C}_{x}\right), \\
\text { subject to } & P_{\text {out }, \mathrm{p}}\left(p_{\mathrm{s}}, \mathcal{C}_{x}\right) \leq P_{\text {out,th }}, \\
& 0 \leq p_{\mathrm{s}} \leq p_{\mathrm{s}, \max }, \\
& 0 \leq \mathcal{C}_{x} \leq 1,
\end{aligned}
$$

\footnotetext{
${ }^{3}$ A similar condition is applied in [20], [21] to maximize the SU instantaneous rate.
}

where $p_{\mathrm{s}, \max }$ is the maximum allowable $\mathrm{SU}$ transmitter power. To solve this problem, we use the PU outage probability expressions derived in the previous section to formulate simpler versions of it. We start by using the PU lower-bound in (25) to relax the optimization problem in (38). Although this relaxation violates the PU constraint, it can lead the reader to understand the trend of the improper signal design in such systems. This idea will be then applied to both the upper bound and the approximate expressions of the PU outage probability which requires complex computations.

1) Lower-bound-based design: Thanks to the simplified PU lower bound expression obtained in (25), the first constraint in (38) can be relaxed in terms of $p_{\mathrm{s}}$ and $\mathcal{C}_{x}$ as, $p_{\mathrm{s}} \leq \mathcal{F}\left(\mathcal{C}_{x}\right)$, where $\mathcal{F}\left(\mathcal{C}_{x}\right)$ is defined as

$$
\mathcal{F}\left(\mathcal{C}_{x}\right)=\frac{\exp \left(-\Psi_{\mathrm{p}}(0)\right)-\left(1-P_{\text {out }, \text { th }}\right)}{\Psi_{\mathrm{p}}\left(\mathcal{C}_{x}\right)\left(1-P_{\text {out }, \text { th }}\right) \overline{\mathcal{I}}_{\mathrm{s}}}
$$

$\mathcal{F}\left(\mathcal{C}_{x}\right)$ represents the maximum allowable SU power that can be used to meet the PU QoS after relaxation, i.e., $P_{\text {out }, \mathrm{p}}^{\mathrm{LB}}\left(p_{\mathrm{s}}, \mathcal{C}_{x}\right)=P_{\text {out,th}}$. Based on (33), one can easily observe that the inequality $\exp \left(-\Psi_{\mathrm{p}}(0)\right)>\left(1-P_{\text {out,th }}\right)$ is always valid, which means that the SU may transmit as long as $\mathcal{I}_{\max }>0$. As a result, the relaxed optimization problem of (38) can be rewritten as

$$
\begin{aligned}
\min _{p_{\mathrm{s}}, \mathcal{C}_{x}} & P_{\text {out }, \mathrm{s}}\left(p_{\mathrm{s}}, \mathcal{C}_{x}\right) \\
\text { subject to } & 0 \leq p_{\mathrm{s}} \leq \min \left(\mathcal{F}\left(\mathcal{C}_{x}\right), p_{\mathrm{s}, \max }\right) \\
& 0 \leq \mathcal{C}_{x} \leq 1
\end{aligned}
$$

Now, one can prove that $\mathcal{F}\left(\mathcal{C}_{x}\right)$ is a monotonically increasing function in $\mathcal{C}_{x}$ (see Appendix B). If $\min \left(\mathcal{F}(0), p_{\mathrm{s}, \max }\right)=$ $p_{\mathrm{s}, \max }$, then the solution is given by (36). On the other hand, when $\min \left(\mathcal{F}\left(\mathcal{C}_{x}\right), p_{\mathrm{s}, \max }\right)=\mathcal{F}\left(\mathcal{C}_{x}\right)$ the problem can be solved by using the monotonic properties of the objective function and the constraints. Since $P_{\text {out,s }}\left(p_{\mathrm{s}}, \mathcal{C}_{x}\right)$ is monotonically decreasing in $p_{\mathrm{s}}$ (See Appendix $\mathrm{C}$ for the proof), then $p_{\mathrm{s}}$ should be assigned the maximum value of the constraint to minimize the SU outage probability, i.e., $p_{\mathrm{s}}=\mathcal{F}\left(\mathcal{C}_{x}\right)$. Therefore, the improper Gaussian signaling optimization problem (40) simplifies to

$$
\begin{aligned}
\min _{\mathcal{C}_{x}} & P_{\text {out }, \mathrm{s}}\left(\mathcal{C}_{x}\right) \\
\text { subject to } & 0 \leq \mathcal{C}_{x} \leq 1, \\
& 0<\mathcal{F}\left(\mathcal{C}_{x}\right) \leq p_{\mathrm{s}, \max },
\end{aligned}
$$

where $P_{\text {out }, \mathrm{s}}\left(\mathcal{C}_{x}\right)=P_{\text {out }, \mathrm{s}}\left(\mathcal{F}\left(\mathcal{C}_{x}\right), \mathcal{C}_{x}\right)$. To solve (41), first we note that $P_{\text {out,s }}\left(\mathcal{C}_{x}\right)$ is monotonically decreasing in $\mathcal{C}_{x}$ as shown in Appendix $\mathrm{D}$, while $\mathcal{F}\left(\mathcal{C}_{x}\right)$ is monotonically increasing in $\mathcal{C}_{x}$. Thus, the SU can achieve lower outage if improper signal with the maximum possible transmitted power of $\mathcal{F}\left(\mathcal{C}_{x}\right)$ is used. As a result, the SU power solution is $p_{\mathrm{s}, \max }$ and the corresponding $\mathcal{C}_{x}$ is given by

$$
\mathcal{C}_{x}= \begin{cases}0 & \text { if } \mathcal{F}(0) \geq p_{\mathrm{s}, \max }, \\ \mathcal{C}_{x}^{*} & \text { if } \mathcal{F}(0)<p_{\mathrm{s}, \max }\end{cases}
$$


where $\mathcal{C}_{x}^{*}$ is the solution of the equation $p_{\mathrm{s}, \max }=\mathcal{F}\left(\mathcal{C}_{x}\right)$ and is expressed as

$$
\mathcal{C}_{x}^{*}=\sqrt{1-\frac{1}{\Gamma_{\mathrm{p}}}\left[\left(p_{\mathrm{p}} \bar{\gamma}_{\mathrm{p}} \Psi_{\mathrm{p}}(0) \frac{p_{\mathrm{s}, \mathrm{PGS}}}{p_{\mathrm{s}, \max }}+1\right)^{2}-1\right]},
$$

where $p_{\mathrm{s}, \mathrm{PGS}}$ is the $\mathrm{SU}$ power computed based on proper Gaussian signaling design (36). The solution tells us that the performance improvement of the improper Gaussian signaling scheme over the proper Gaussian signaling scheme occurs when the SU increases its transmitted power. To summarize, the benefits of employing the improper signaling scheme exists if $\mathcal{I}_{\max }>0$ and $\mathcal{F}(0)<p_{\mathrm{s}, \max }$ are satisfied simultaneously. In addition, the benefit of using such a scheme increases with the increase of $\overline{\mathcal{I}}_{\mathrm{s}}$. In this case, the SU with the proper Gaussian signaling scheme reduces its transmitted power to meet the PU QoS, while switching to improper Gaussian signaling allows the SU to increase its power without affecting the PU QoS.

The aforementioned lower-bound-based solution shows that $\mathcal{C}_{x}$ goes to 1 with the increase of the available power budget as can be observed from (43). However, this result may violate the PU QoS especially for large $\overline{\mathcal{I}}_{\mathrm{s}}$ and very small $\exp \left(-\Psi_{\mathrm{p}}(0)\right)-\left(1-P_{\text {out }, \text { th }}\right)$, i.e., $\mathcal{I}_{\max }$ is very small . In order to avoid such violations on the PU, we adopt the upper bound outage probability derived in Section III to design $p_{\mathrm{s}}$ and $\mathcal{C}_{x}$ in the following subsection.

2) Upper-bound-based design: To provide a robust design of the SU signal parameters, we need to use the upper bound outage probability expression in the design process to satisfy the outage probability constraint in (38). As such, the upper bound expression can be used to simplify the new overfilled design problem by imposing $P_{\text {out, }}^{\mathrm{UB}}\left(p_{\mathrm{s}}, \mathcal{C}_{x}\right)=P_{\text {out,th }}$. In this problem, $p_{\mathrm{s}}$ can not be expressed in terms of $\mathcal{C}_{x}$, while $\mathcal{C}_{x}$ can be expressed in terms of $p_{\mathrm{s}}$ as

$\mathcal{C}_{x}^{\mathrm{UB}}\left(p_{\mathrm{s}}\right)=\sqrt{\left(\frac{\alpha_{\mathrm{UB}}+1}{\alpha_{\mathrm{UB}}}\right)^{2}\left(1-\frac{\Lambda_{\mathrm{UB}}\left(p_{\mathrm{s}}\right)}{\Gamma_{\mathrm{p}}}\left[2+\Lambda_{\mathrm{UB}}\left(p_{\mathrm{s}}\right)\right]\right)}$

where $\Lambda_{\mathrm{UB}}\left(p_{\mathrm{s}}\right)$ is defined as

$$
\begin{aligned}
\Lambda_{\mathrm{UB}}\left(p_{\mathrm{s}}\right)= & \frac{p_{\mathrm{p}} \bar{\gamma}_{\mathrm{p}}}{\alpha_{\mathrm{UB}}+1} \mathbb{W}\left\{\frac{\left(\alpha_{\mathrm{UB}}+1\right) \exp \left(\frac{1}{\overline{\mathcal{I}}_{\mathrm{s}} p_{\mathrm{s}}}\right)}{\overline{\mathcal{\mathcal { I }}}_{\mathrm{s}} p_{\mathrm{s}}\left(1-P_{\text {out }, \text { th }}-\mu_{\mathrm{UB}}\left(p_{\mathrm{s}}\right)\right)}\right\} \\
& -\frac{p_{\mathrm{p}} \bar{\gamma}_{\mathrm{p}}}{\overline{\overline{\mathcal{I}}}_{\mathrm{s}} p_{\mathrm{s}}},
\end{aligned}
$$

with $\mathbb{W}\{$.$\} is defined as the Lambert-W function [28], and$ $\mu_{\mathrm{UB}}\left(p_{\mathrm{s}}\right)$ is defined as

$$
\begin{aligned}
\mu_{\mathrm{UB}}\left(p_{\mathrm{s}}\right)=\frac{\exp \left(-\Psi_{\mathrm{p}}(0)\right)}{1+\overline{\mathcal{I}}_{\mathrm{s}} p_{\mathrm{s}} \Psi_{\mathrm{p}}(0)} & \\
& -\frac{\exp \left(-\frac{\alpha_{\mathrm{UB}}}{\overline{\mathcal{I}}_{\mathrm{s}} p_{\mathrm{s}}}-\left(\alpha_{\mathrm{UB}}+1\right) \Psi_{\mathrm{p}}(0)\right)}{1+\overline{\mathcal{I}}_{\mathrm{s}} p_{\mathrm{s}} \Psi_{\mathrm{p}}(0)} .
\end{aligned}
$$

To compute the signal parameters that minimize the SU upper bound outage probability, we consider the following simplified optimization problem to compute $p_{\mathrm{s}}$

$$
\begin{aligned}
\min _{p_{\mathrm{s}}} & P_{\mathrm{out}, \mathrm{s}}^{\mathrm{UB}}\left(p_{\mathrm{s}}\right) \\
\text { subject to } & 0 \leq \mathcal{C}_{x}^{\mathrm{UB}}\left(p_{\mathrm{s}}\right) \leq 1, \quad 0<p_{\mathrm{s}} \leq p_{\mathrm{s}, \max },
\end{aligned}
$$

where $P_{\text {out }, \mathrm{s}}^{\mathrm{UB}}\left(p_{\mathrm{s}}\right)=P_{\text {out }, \mathrm{s}}\left(p_{\mathrm{s}}, \mathcal{C}_{x}^{\mathrm{UB}}\left(p_{\mathrm{s}}\right)\right)$ then we can find $\mathcal{C}_{x}^{\mathrm{UB}}$ from (44).

Unfortunately, the optimization problem in (47) is not simple compared with the lower-bound based optimization problem (41) solved in the previous subsection, which has some interesting monotonic characteristics. On the other hand, we can not prove similar characteristics in the upper-bound based problem. However, inspired by the solution trend of the previous problem, the $\mathrm{SU}$ power allocation can be expressed as

$$
p_{\mathrm{s}}^{*}= \begin{cases}p_{\mathrm{s}, \max } & \text { if } p_{\mathrm{s}}^{\mathrm{UB}}(0) \geq p_{\mathrm{s}, \max }, \\ p_{\mathrm{s}, \max } & \text { if } p_{\mathrm{s}}^{\mathrm{UB}}(0)<p_{\mathrm{s}, \max } \text { and } \mathcal{C}_{x}^{\mathrm{UB}}\left(p_{\mathrm{s}, \max }\right)<1, \\ p_{\mathrm{s}}^{\mathrm{UB}}(1) & \text { if } p_{\mathrm{s}}^{\mathrm{UB}}(1)<p_{\mathrm{s}, \max } \text { and } \mathcal{C}_{x}^{\mathrm{UB}}\left(p_{\mathrm{s}, \max }\right) \geq 1,\end{cases}
$$

where $p_{\mathrm{s}}^{\mathrm{UB}}($.$) is the inverse function of (44) and is evaluated$ numerically. Then, the corresponding $\mathcal{C}_{x}$ is computed from

$$
\mathcal{C}_{x}^{*}= \begin{cases}0 & \text { if } p_{\mathrm{s}}^{\mathrm{UB}}(0) \geq p_{\mathrm{s}, \max }, \\ \mathcal{C}_{x}^{\mathrm{UB}}\left(p_{\mathrm{s}, \max }\right) & \text { if } p_{\mathrm{s}}^{\mathrm{UB}}(0)<p_{\mathrm{s}, \max } \text { and } \mathcal{C}_{x}^{\mathrm{UB}}\left(p_{\mathrm{s}, \max }\right)<1, \\ 1 & \text { if } p_{\mathrm{s}}^{\mathrm{UB}}(0)<p_{\mathrm{s}, \max } \text { and } \mathcal{C}_{x}^{\mathrm{UB}}\left(p_{\mathrm{s}, \max }\right) \geq 1,\end{cases}
$$

3) Approximate outage probability based design: In this subsection, we use the PU approximate expression evaluated at $\alpha_{\mathrm{UB}}$ to obtain a tight approximation to the exact PU outage probability. Although, we can not guarantee that the approximate expression provides upper or lower bound approximation, we want to have an idea about the benefits that can be reaped from the improper Gaussian signaling in our proposed model. First we relax the PU outage probability in (38), similar to the upper bound based design, by imposing $\tilde{P}_{\text {out }, \mathrm{p}}\left(p_{\mathrm{s}}, \mathcal{C}_{x}, \alpha_{\mathrm{UB}}\right)=P_{\text {out,th }}$. After this relaxation, the PU outage probability constraint can be rewritten in terms of $\mathcal{C}_{x}^{*}$ that is expressed in terms of $p_{\mathrm{s}}$ as

$$
\mathcal{C}_{x}^{\mathrm{Ap}}\left(p_{\mathrm{s}}\right)=\sqrt{1-\frac{\Lambda_{\mathrm{Ap}}\left(p_{\mathrm{s}}\right)}{\Gamma_{\mathrm{p}}}\left(2+\Lambda_{\mathrm{Ap}}\left(p_{\mathrm{s}}\right)\right)},
$$

where $\Lambda_{\mathrm{Ap}}\left(p_{\mathrm{s}}\right)$ is defined as

$$
\Lambda_{\mathrm{Ap}}\left(p_{\mathrm{s}}\right)=\frac{p_{\mathrm{p}} \bar{\gamma}_{\mathrm{p}}}{\alpha_{\mathrm{UB}}} \mathbb{W}\left\{\frac{\alpha_{\mathrm{UB}} /\left(\overline{\mathcal{I}}_{\mathrm{s}} p_{\mathrm{s}}\right)}{1-P_{\mathrm{out}, \mathrm{th}}-\mu_{\mathrm{UB}}\left(p_{\mathrm{s}}\right)}\right\}-\frac{p_{\mathrm{p}} \bar{\gamma}_{\mathrm{p}}}{\overline{\mathcal{I}}_{\mathrm{s}} p_{\mathrm{s}}},
$$

and $\mu_{\mathrm{Ap}}\left(p_{\mathrm{s}}\right)$ is defined as

$$
\mu_{\mathrm{Ap}}\left(p_{\mathrm{s}}\right)=\frac{\exp \left(-\Psi_{\mathrm{p}}(0)\right)}{1+p_{\mathrm{s}} \overline{\mathcal{I}}_{\mathrm{s}} \Psi_{\mathrm{p}}(0)}-\frac{\exp \left(-\frac{\alpha_{\mathrm{UB}}}{p_{\mathrm{s}} \overline{\mathcal{I}}_{\mathrm{s}}}-\alpha_{\mathrm{UB}} \Psi_{\mathrm{p}}(0)\right)}{1+p_{\mathrm{s}} \overline{\mathcal{I}}_{\mathrm{s}} \Psi_{\mathrm{p}}(0)} .
$$

As can be seen from $(50), \mathcal{C}_{x}^{\mathrm{Ap}}\left(p_{\mathrm{s}}\right)$ has a similar expression to the upper bound based design, which makes the optimization problem non-tractable. Thus, we follow the same upper bound based solution and use the approximate expression in (50) to find $p_{\mathrm{s}}$ and $\mathcal{C}_{x}$ from (48) and (49). 
TABLE I

SIMULATION PARAMETERS.

\begin{tabular}{|c|c|c|}
\hline $\mathcal{I}_{\max }=2 \mathrm{~dB}$ & $p_{\mathrm{s}, \max }=1 \mathrm{~W}$ & $P_{\text {out th }}=0.01$ \\
\hline$R_{0, \mathrm{p}}=1 \mathrm{~b} / \mathrm{s} / \mathrm{Hz}$ & $R_{0, \mathrm{~s}}=1 \mathrm{~b} / \mathrm{s} / \mathrm{Hz}$ & $\mathcal{C}_{x}=0.8$ \\
\hline$\overline{\mathcal{I}}_{\mathrm{s}}=5 \mathrm{~dB}$ & $\overline{\mathcal{I}}_{\mathrm{p}}=3 \mathrm{~dB}$ & $\bar{\gamma}_{\mathrm{s}}=20 \mathrm{~dB}$ \\
\hline $\bar{\gamma}_{\mathrm{p}}=20 \mathrm{~dB}$ & $p_{\mathrm{p}}=1 \mathrm{~W}$ & \\
\hline
\end{tabular}

As for the computation complexity, the approximate based design has the same complexity as the upper bound based design. Both methods compute the circularity coefficient using the evaluation of Lambert-W function. In addition, the computation of $p_{\mathrm{s}}$ at $\mathcal{C}_{x}=1$ needs the numerical inverse evaluation of (44) or (50) for the upper bound or the approximate expression based methods, respectively. Each expression needs to compute the Lambert- $\mathrm{W}$ function several times according to number of iterations used to evaluate the inverse function. For example, if we use the bisection method in numerical computation, then the complexity is $\mathcal{O}\left(\log _{2} \frac{\Delta \lambda}{\epsilon}\right)$, where $\Delta \lambda$ is the interval range that captures the solution and $\epsilon$ is the solution accuracy [29]. On the other hand, the computation of $\mathcal{C}_{x}$ in the lower bound based design has a closed form expression expressed in (43).

\section{Numerical Results}

In this section, we provide numerical results to assess the accuracy of the derived PU outage probability expressions and compare between different design methods. The comparison is done by monitoring both the gain of the SU outage probability performance and the fulfillment of the PU outage probability. After showing that the improper Gaussian signaling system design based on the approximate outage probability gives acceptable performance with respect to other bounds, we compare it with proper Gaussian signaling system design. Throughout this comparison, we highlight the effects of different system parameters on the benefit of the improper Gaussian signaling based system over the proper Gaussian signaling based system. In the following subsections, we use the simulation parameters listed in Table 1, unless otherwise specified.

\section{A. PU outage probability}

To investigate the impact of different parameters on the tightness of the upper bound, the lower bound, the approximate expressions to the exact one, we introduce the following simulation examples.

Example 1: In the first example, we study the PU outage probability versus $\bar{\gamma}_{\mathrm{p}}$ assuming different SU interference in Fig. 3. At low $\overline{\mathcal{I}}_{\mathrm{s}}$, the upper bound is found to be closer to the exact PU outage probability than the lower bound. As $\overline{\mathcal{I}}_{\mathrm{s}}$ increases, the gap between the upper bound and the exact PU outage probability increases. With further increment of $\overline{\mathcal{I}}_{\mathrm{s}}$, the situation of the lower and the upper bounds with respect to the exact PU outage probability reverses, where the lower bound becomes tighter to the exact PU outage probability than the upper bound. When it comes to the approximate PU outage probability, $\alpha$ is evaluated from the optimal upper bound condition in (29). The approximate expression represents almost

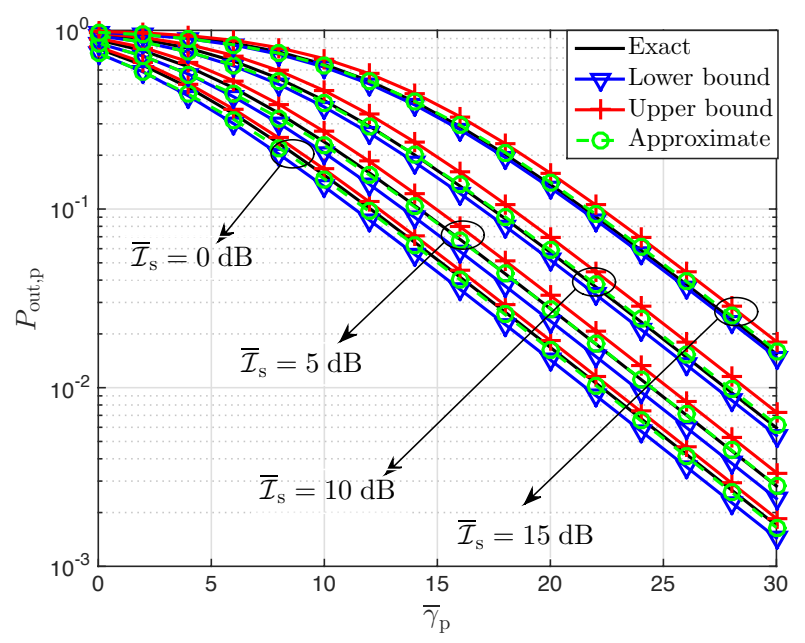

Fig. 3. A comparison between the exact PU outage probability, the lower bound, the upper bound and the approximate expressions versus $\bar{\gamma}_{\mathrm{p}}$ for $\overline{\mathcal{I}}_{\mathrm{s}}=$ $0,5,10,15 \mathrm{~dB}$.

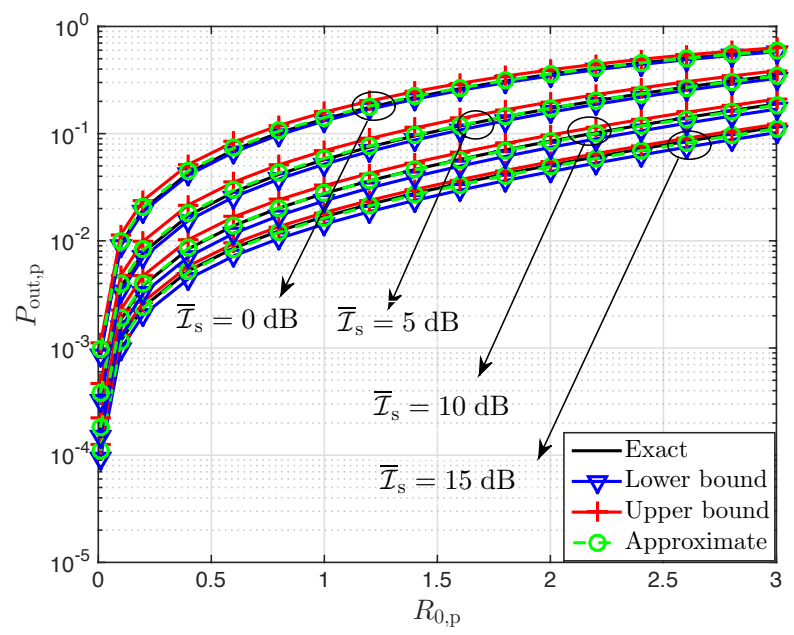

Fig. 4. A comparison between the exact PU outage probability, the lower bound, the upper bound and the approximate expressions versus $R_{0, \mathrm{p}}$ for $\overline{\mathcal{I}}_{\mathrm{s}}=0,5,10,15 \mathrm{~dB}$.

the same performance of the exact PU outage probability for different $\bar{\gamma}_{\mathrm{p}}$ and $\overline{\mathcal{I}}_{\mathrm{s}}$.

Example 2: In this example, we evaluate the performance of different PU outage probability expressions versus $R_{0, \mathrm{p}}$ for different $\overline{\mathcal{I}}_{\mathrm{s}}$ in Fig. 4. At very low values of $R_{0, \mathrm{p}}$, the upper bound, lower bound and the approximate PU outage probability provide nearly the same performance as the PU exact outage probability expression. The same observation can be observed at high $R_{0, \mathrm{p}}$ and low $\overline{\mathcal{I}}_{\mathrm{s}}$, i.e., $\overline{\mathcal{I}}_{\mathrm{s}}=0 \mathrm{~dB}$. At the other ranges of $R_{0, \mathrm{p}}$ and $\overline{\mathcal{I}}_{\mathrm{s}}$, the relative performance of different PU outage probability expressions are the same as in simulation example 1.

Example 3: The third simulation example compares the PU outage probability expressions with the exact PU outage probability versus $\mathcal{C}_{x}$ for different $R_{0, \mathrm{p}}$ in Fig. 5. All the PU outage probability expressions achieve nearly the same performance as the exact PU outage probability for $\mathcal{C}_{x} \leq 0.6$. 


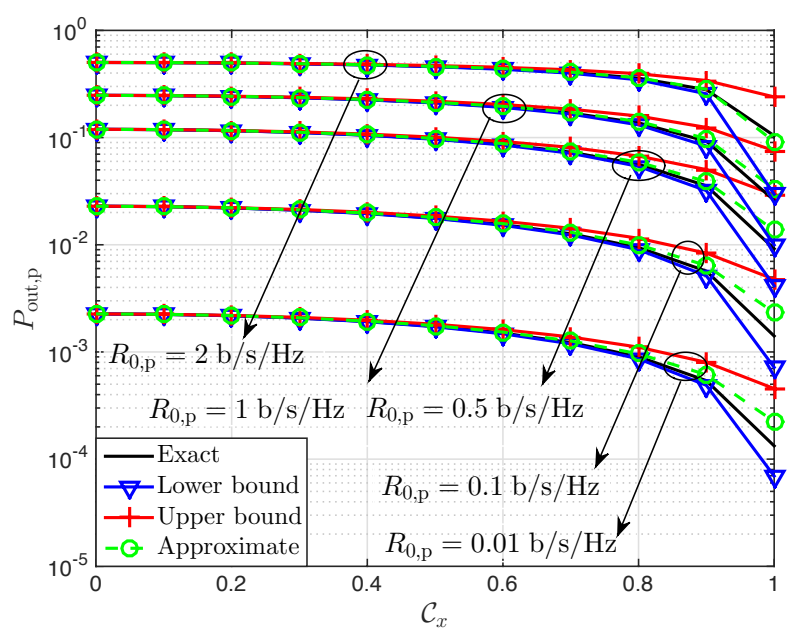

Fig. 5. A comparison between the exact PU outage probability and the lower bound, the upper bound and the approximate expression versus $\mathcal{C}_{x}$ for different $R_{0, \mathrm{p}}$.

For $0.6<\mathcal{C}_{x} \leq 1$, the lower bound becomes closer to the exact PU outage probability than the upper bound. Similar to the previous examples, the approximate PU outage probability gives a close performance to the exact outage probability for different $R_{0, \mathrm{p}}$.

\section{B. Comparison between the Design Methods}

In this subsection, we compare between the SU performance improvement using the proper Gaussian design and the improper Gaussian design based on the lower bound, the upper bound, and the approximate PU outage probability expressions. In addition, we monitor the fulfillment of the PU performance by plotting the PU outage probability versus $\bar{\gamma}_{\mathrm{p}}$. The PU outage probability expression is evaluated numerically using (21) based on the signal parameters obtained from different design methods.

Example 4: Under the assumption of $\overline{\mathcal{I}}_{\mathrm{s}}=5 \mathrm{~dB}$, we observe that the improper Gaussian signaling system achieves better performance than the proper Gaussian signaling system at medium and high $\bar{\gamma}_{\mathrm{p}}$ values as shown in Fig. 6. The performance improvement increases as the PU channel improves because having good PU channel permits using more power at the SU side. Both the upper bound based design and the approximate based design achieve nearly the same performance, while the lower bound based design achieves a slight better performance than other methods at an expected violation of the PU outage as can be observed in Fig. 6. On the other hand, both the upper bound and approximate based designs meet the PU outage probability QoS requirement.

Example 5: In this example, we assume higher SU interference on the PU, where $\overline{\mathcal{I}}_{\mathrm{s}}=10 \mathrm{~dB}$. We observe that the impact of high $\overline{\mathcal{I}}_{\mathrm{s}}$ on both the PU and the SU becomes different from the low $\overline{\mathcal{I}}_{\mathrm{s}}$ case, as can be seen in Fig. 7. First, from the SU side, the best performance is achieved by the lower bound based design but at a violation cost of the PU outage performance. As for the approximate based
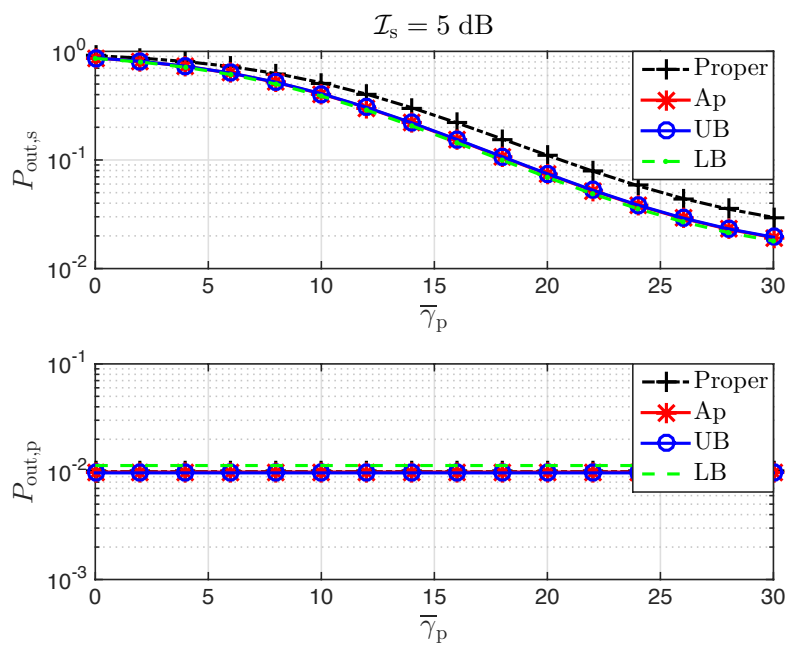

Fig. 6. A comparison between the SU outage probability that uses proper Gaussian design, lower bound based design, the upper bound based design, and the approximate based design at $\overline{\mathcal{I}}_{\mathrm{s}}=5 \mathrm{~dB}$.
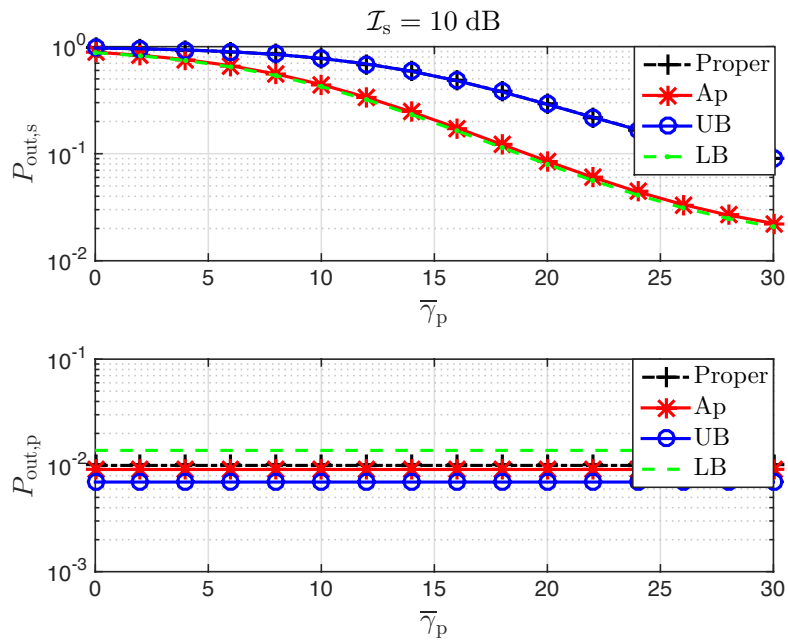

Fig. 7. A comparison between the SU outage probability that uses the proper Gaussian based design, lower bound PU outage probability based design, upper bound based design, and the approximate based design at $\overline{\mathcal{I}}_{\mathrm{s}}=10 \mathrm{~dB}$.

design, it achieves a very close performance to the lower bound based design while meeting PU outage constraints. On the other hand, the performance of the upper bound based design degrades and achieves the same performance as the SU outage probability of the proper Gaussian system design. Interestingly, the upper bound based design reaps the improper Gaussian design benefit through a further protection of the PU outage probability, as shown in Fig. 7. Thus, in order to investigate the benefit of improper versus proper on the SU outage, we adopt the approximate based design in the following subsection.

\section{Improper versus Proper Gaussian Signaling}

In this subsection, we explore the role of adopting improper Gaussian signaling in improving the underlay cognitive radio performance. For this purpose, we introduce different simulation examples to investigate the impact of system parameters 


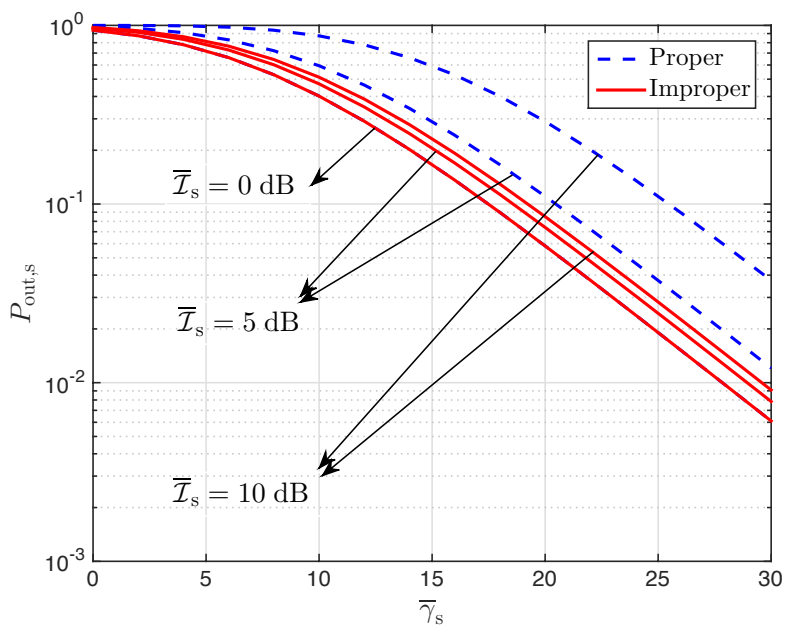

Fig. 8. SU outage probability for the proper and the improper Gaussian signaling schemes versus $\bar{\gamma}_{\mathrm{s}}$ assuming different $\overline{\mathcal{I}}_{\mathrm{s}}$.

in reaping the benefits of adopting the improper Gaussian signaling. Throughout the following numerical results, we compare the SU outage probability of both schemes proper and improper Gaussian signaling based on the approximate PU outage probability.

Example 6: Fig. 8 plots the SU outage probability for both improper and proper Gaussian signaling systems versus $\bar{\gamma}_{\mathrm{s}}$ assuming different values of $\overline{\mathcal{I}}_{\mathrm{s}}$. At low SU interference levels on the PU, i.e., $\overline{\mathcal{I}}_{\mathrm{s}}=0 \mathrm{~dB}$, the proper Gaussian signaling system tends to use its maximum power budget, thus the improper Gaussian signaling scheme reduces to the proper one. As $\overline{\mathcal{I}}_{\mathrm{s}}$ increases, the proper Gaussian signaling system uses less power to satisfy the PU QoS. On the other hand, the improper Gaussian signaling system uses more power and compensates its impact on the PU by increasing $\mathcal{C}_{x}$. Interestingly, the proper Gaussian signaling scheme degrades significantly with increasing $\overline{\mathcal{I}}_{\mathrm{s}}$, while the improper Gaussian signaling scheme has immunity against $\overline{\mathcal{I}}_{\mathrm{s}}$. As for $\bar{\gamma}_{\mathrm{s}}$, both the proper and the improper Gaussian signaling schemes improves with the increase of $\bar{\gamma}_{\mathrm{s}}$.

Example 7: In this example, we study the SU outage probability for both signaling schemes versus $\overline{\mathcal{I}}_{\mathrm{p}}$ assuming different $R_{0, \mathrm{p}}$ values at $\overline{\mathcal{I}}_{\mathrm{s}}=5 \mathrm{~dB}$, as shown in Fig. 9. First, we observe that the improper Gaussian signaling system has a room for improvement over the proper signaling system because of the moderate value of SU interference on the PU. Second, the improvement decreases with the increase of $\overline{\mathcal{I}}_{\mathrm{p}}$, where the PU interference channel impact becomes dominant in the SU outage performance. Finally, low PU rate requirements, allows creating more coverage opportunities for the SU especially when the improper Gaussian signaling system is adopted. In conclusion, as the PU rate requirement decreases and/or the PU interference impact on the SU decreases, the improper Gaussian signaling system has increased benefits over the proper one.

Example 8: In this example, we study the joint impact of $R_{0, \mathrm{~s}}$ and $\overline{\mathcal{I}}_{\mathrm{s}}$ on the improvement performance of improper Gaussian signaling system over the proper Gaussian signaling

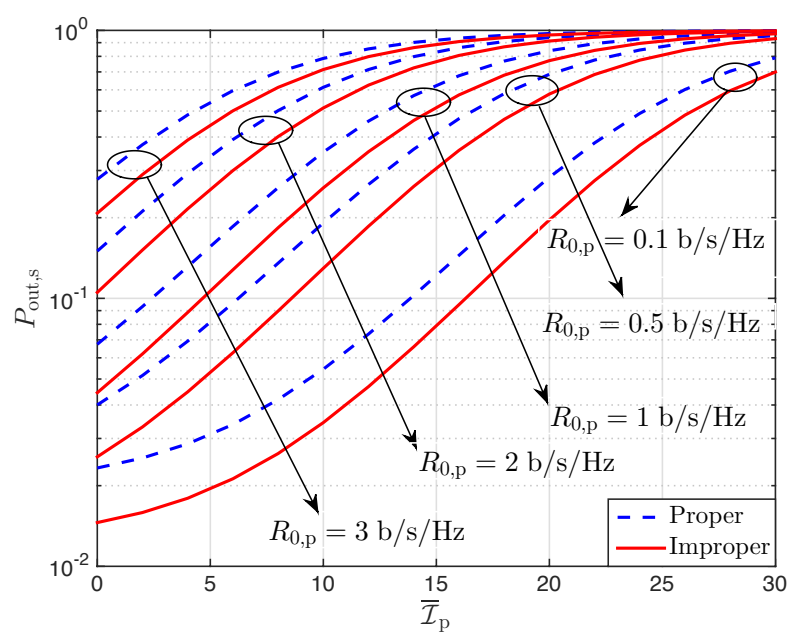

Fig. 9. SU outage probability for the proper and the improper Gaussian signaling versus $\overline{\mathcal{I}}_{\mathrm{p}}$ for different $R_{0, \mathrm{p}}$.

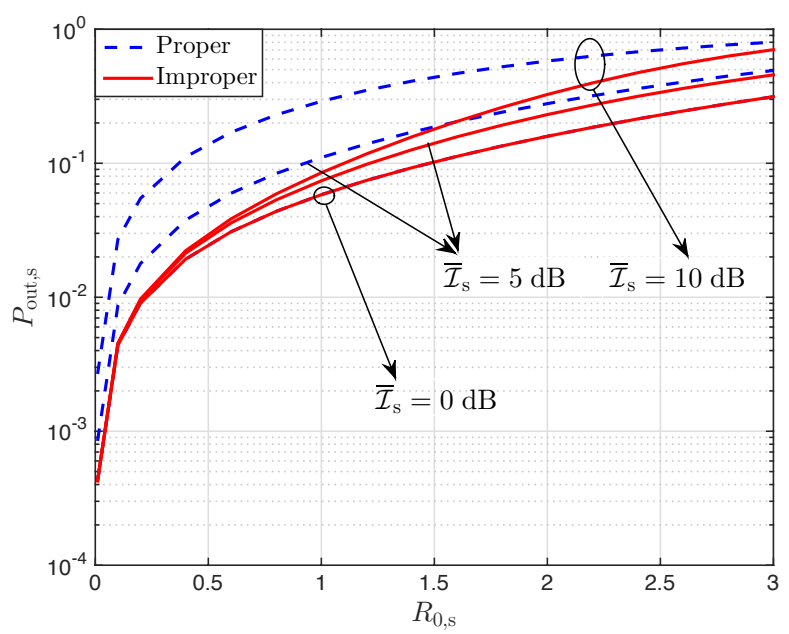

Fig. 10. The SU outage probability for the proper and the improper Gaussian signaling schemes versus $R_{0, \mathrm{~s}}$ for different $\overline{\mathcal{I}}_{\mathrm{s}}$.

system as shown in Fig. 10. Similar to the earlier results, very low $\overline{\mathcal{I}}_{\mathrm{s}}$ makes both proper and improper Gaussian signaling systems have the same performance, while the improvement occurs at high $\overline{\mathcal{I}}_{\mathrm{s}}$ values. As for the SU rate impact, the improvement is observed to be in the middle values range of the $\mathrm{SU}$ rate at $\overline{\mathcal{I}}_{\mathrm{s}}=5,10 \mathrm{~dB}$.

\section{CONCLUSION}

In this paper, we studied the outage probability of underlay cognitive radio system with improper Gaussian signaling scheme. We derived closed form expression for the SU outage probability and tight bounds for the PU. Based on the derived expressions and using the average CSI, we adjusted the SU power and circularity coefficient to improve its performance measured in terms of the outage probability while satisfying the PU QoS and meeting the SU power budget. For many practical scenarios that we considered in this paper, our simulation results show that the benefit of improper Gaussian 
signaling scheme over the proper Gaussian signaling increases as the interference-to-noise ratio of the SU to the PU increases for a specific SU target rate.

\section{APPENDIX A \\ PROOF OF $\tilde{P}_{\text {out }, \mathrm{p}}\left(p_{\mathrm{s}}, \mathcal{C}_{x}, \alpha\right) \leq P_{\text {out }, \mathrm{p}}^{\mathrm{UB}}\left(p_{\mathrm{s}}, \mathcal{C}_{x}, \alpha\right)$}

First, one can show easily that $\Psi_{\mathrm{p}}\left(\frac{\alpha x}{\alpha+1}\right)$ is monotonically decreasing in $\alpha$, thus we have

$$
\frac{1}{1+p_{\mathrm{s}} \overline{\mathcal{I}}_{\mathrm{s}} \Psi_{\mathrm{p}}\left(\frac{\mathcal{C}_{x} \alpha}{1+\alpha}\right)}<\frac{1}{1+\overline{\mathcal{I}}_{\mathrm{s}} p_{\mathrm{s}} \Psi_{\mathrm{p}}\left(\mathcal{C}_{x}\right)}
$$

and $(1+\alpha) \Psi_{\mathrm{p}}\left(\frac{\mathcal{C}_{x} \alpha}{1+\alpha}\right) \geq \alpha \Psi_{\mathrm{p}}\left(\mathcal{C}_{x}\right)$ for $\alpha \geq 0$, which results in

$$
\exp \left(-(\alpha+1) \Psi\left(\frac{\mathcal{C}_{x} \alpha}{1+\alpha}\right)\right)<\exp \left(-\alpha \Psi\left(\mathcal{C}_{x}\right)\right) .
$$

From (53) and (54), we obtain

$$
\frac{\exp \left(-\alpha \Psi\left(\mathcal{C}_{x}\right)\right)}{1+\overline{\mathcal{I}}_{\mathrm{s}} p_{\mathrm{s}} \Psi_{\mathrm{p}}\left(\mathcal{C}_{x}\right)}>\frac{\exp \left(-(\alpha+1) \Psi\left(\frac{\mathcal{C}_{x} \alpha}{1+\alpha}\right)\right)}{1+p_{\mathrm{s}} \overline{\mathcal{I}}_{\mathrm{s}} \Psi_{\mathrm{p}}\left(\frac{\mathcal{C}_{x} \alpha}{1+\alpha}\right)}
$$

Based on the monotonicity characteristic of $\Psi_{\mathrm{p}}(x)$ in $\alpha$, we obtain

$$
-\frac{\exp \left(-(\alpha+1) \Psi_{\mathrm{p}}(0)\right)}{1+p_{\mathrm{s}} \overline{\mathcal{I}}_{\mathrm{s}} \Psi_{\mathrm{p}}(0)}>-\frac{\exp \left(-\alpha \Psi_{\mathrm{p}}(0)\right)}{1+p_{\mathrm{s}} \overline{\mathcal{I}}_{\mathrm{s}} \Psi_{\mathrm{p}}(0)} .
$$

By adding (55) and (56), we obtain

$$
\begin{array}{r}
\frac{\exp \left(-(\alpha+1) \Psi_{\mathrm{p}}(0)\right)}{1+p_{\mathrm{s}} \overline{\mathcal{I}}_{\mathrm{s}} \Psi_{\mathrm{p}}(0)}-\frac{\exp \left(-\alpha \Psi_{\mathrm{p}}(0)\right)}{1+p_{\mathrm{s}} \overline{\mathcal{I}}_{\mathrm{s}} \Psi_{\mathrm{p}}(0)}> \\
\frac{\exp \left(-(\alpha+1) \Psi_{\mathrm{p}}\left(\frac{\mathcal{C}_{x} \alpha}{1+\alpha}\right)\right)}{1+p_{\mathrm{s}} \overline{\mathcal{I}}_{\mathrm{s}} \Psi_{\mathrm{p}}\left(\frac{\mathcal{C}_{x} \alpha}{1+\alpha}\right)}-\frac{\exp \left(-\alpha \Psi_{\mathrm{p}}\left(\mathcal{C}_{x}\right)\right)}{1+\overline{\mathcal{I}}_{\mathrm{s}} p_{\mathrm{s}} \Psi_{\mathrm{p}}\left(\mathcal{C}_{x}\right)},
\end{array}
$$

which proves that $\tilde{P}_{\text {out }, \mathrm{p}}\left(p_{\mathrm{s}}, \mathcal{C}_{x}, \alpha\right) \leq P_{\text {out }, \mathrm{p}}^{\mathrm{UB}}\left(p_{\mathrm{s}}, \mathcal{C}_{x}, \alpha\right)$.

\section{APPENDIX B}

PROOF OF THE MONOTONICALLY INCREASING CHARACTERISTICS OF $\mathcal{F}\left(\mathcal{C}_{x}\right)$ IN $\mathcal{C}_{x}$

$$
\frac{\partial p_{\mathrm{s}}\left(\mathcal{C}_{x}\right)}{\partial \mathcal{C}_{x}}=-\frac{\exp \left(-\Psi_{\mathrm{p}}(0)\right)-\left(1-P_{\text {out th }}\right)}{\Psi_{\mathrm{p}}^{2}\left(\mathcal{C}_{x}\right)\left(1-P_{\text {out }, \text { th }}\right) \overline{\mathcal{I}}_{\mathrm{s}}} \times \frac{\partial \Psi_{\mathrm{p}}\left(\mathcal{C}_{x}\right)}{\partial \mathcal{C}_{x}}
$$

where $\frac{\partial \Psi_{\mathrm{p}}\left(\mathcal{C}_{x}\right)}{\partial \mathcal{C}_{x}}$ is found to be expressed as

$$
\frac{\partial \Psi_{\mathrm{p}}\left(\mathcal{C}_{x}\right)}{\partial \mathcal{C}_{x}}=-\frac{\Gamma_{\mathrm{p}} \mathcal{C}_{x}}{p_{\mathrm{p}} \bar{\gamma}_{\mathrm{p}} \sqrt{1+\Gamma_{\mathrm{p}}\left[1-\mathcal{C}_{x}^{2}\right]}}
$$

Since $\frac{\partial \Psi_{\mathrm{p}}\left(\mathcal{C}_{x}\right)}{\partial \mathcal{C}_{x}} \leq 0$, then $\frac{\partial p_{\mathrm{s}}\left(\mathcal{C}_{x}\right)}{\partial \mathcal{C}_{x}} \geq 0$, as long as

$$
\exp \left(-\Psi_{\mathrm{p}}(0)\right)>\left(1-P_{\text {out }, \text { th }}\right),
$$

which is valid as long as the maximum marginal interferenceto-noise ratio does not equal zero, i.e., $\mathcal{I}_{\max }>0$, otherwise, the SU remains silent. Thus, $p_{\mathrm{s}}\left(\mathcal{C}_{x}\right)$ is monotonically increasing in $\mathcal{C}_{x}$.

\section{APPENDIX C}

PROOF OF THE MONOTONIC DECREASING CHARACTERISTIC OF $P_{\text {out }, \mathrm{s}}\left(p_{\mathrm{s}}, \mathcal{C}_{x}\right)$ With RESPECT TO $p_{\mathrm{s}}$

First, we find the first derivative of $P_{\text {out }, \mathrm{s}}\left(p_{\mathrm{s}}, \mathcal{C}_{x}\right)$ with respect to $p_{\mathrm{s}}$

$$
\begin{gathered}
\frac{\partial P_{\text {out }, \mathrm{s}}\left(p_{\mathrm{s}}, \mathcal{C}_{x}\right)}{\partial p_{\mathrm{s}}}=\frac{1}{1-\mathcal{C}_{x}^{2}+\overline{\mathcal{I}}_{\mathrm{p}} p_{\mathrm{p}} \Psi_{\mathrm{s}}\left(\mathcal{C}_{x}\right)} \frac{\partial \Psi_{\mathrm{s}}\left(\mathcal{C}_{x}\right)}{\partial p_{\mathrm{s}}} \times \\
\left(1+\frac{\left(1-\mathcal{C}_{x}^{2}\right) \overline{\mathcal{I}}_{\mathrm{p}} p_{\mathrm{p}}}{1-\mathcal{C}_{x}^{2}+\overline{\mathcal{I}}_{\mathrm{p}} p_{\mathrm{p}} \Psi_{\mathrm{s}}\left(\mathcal{C}_{x}\right)}\right) \exp \left(-\frac{\Psi_{\mathrm{s}}\left(\mathcal{C}_{x}\right)}{1-\mathcal{C}_{x}^{2}}\right),
\end{gathered}
$$

where $\frac{\partial \Psi_{\mathrm{s}}\left(\mathcal{C}_{x}\right)}{\partial p_{\mathrm{s}}}$ can be found from

$$
\frac{\partial \Psi_{\mathrm{s}}\left(\mathcal{C}_{x}\right)}{\partial p_{\mathrm{s}}}=-\frac{\sqrt{1+\Gamma_{\mathrm{s}}\left(1-\mathcal{C}_{x}^{2}\right)}-1}{p_{\mathrm{s}}^{2} \bar{\gamma}_{\mathrm{s}}} .
$$

Since $\frac{\partial \Psi_{\mathrm{s}}\left(\mathcal{C}_{x}\right)}{\partial p_{\mathrm{s}}} \leq 0$, then $\frac{\partial P_{\mathrm{out}, \mathrm{s}}\left(p_{\mathrm{s}}, \mathcal{C}_{x}\right)}{\partial p_{\mathrm{s}}} \leq 0$, which proves that $P_{\text {out }, \mathrm{s}}\left(p_{\mathrm{s}}, \mathcal{C}_{x}\right)$ is monotonically decreasing in $p_{\mathrm{s}}$.

\section{APPENDIX D}

PROOF OF THE MONOTONICALLY DECREASING CHARACTERISTICS OF $P_{\text {out }, \mathrm{s}}\left(\mathcal{C}_{x}\right)$

First we express $P_{\text {out }, \mathrm{s}}\left(\mathcal{C}_{x}\right)$ as

$$
\begin{aligned}
P_{\text {out }, \mathrm{s}}\left(\mathcal{C}_{x}\right) & =P_{\text {out }, \mathrm{s}}(g, y) \\
& =1-\frac{g y}{g y+\overline{\mathcal{I}}_{\mathrm{p}} p_{\mathrm{p}}} \exp \left(-\frac{1}{g y}\right),
\end{aligned}
$$

where $g\left(\mathcal{C}_{x}\right)$ and $y\left(\mathcal{C}_{x}\right)$ are defined as

$$
\begin{array}{r}
g\left(\mathcal{C}_{x}\right)=\bar{\gamma}_{\mathrm{s}} \sqrt{1-\mathcal{C}_{x}^{2}} p_{\mathrm{s}}\left(\mathcal{C}_{x}\right), \\
y\left(\mathcal{C}_{x}\right)=\frac{\sqrt{1-\mathcal{C}_{x}^{2}}}{\sqrt{1+\Gamma_{\mathrm{s}}\left(1-\mathcal{C}_{x}^{2}\right)}-1} .
\end{array}
$$

Now, we test the behavior of $g\left(\mathcal{C}_{x}\right)$ through the evaluation of $\frac{\partial P_{\text {out }, \mathrm{s}}(g, y)}{\partial g}$, which is found to be

$$
\begin{aligned}
& \frac{\partial P_{\mathrm{out}, \mathrm{s}}(g, y)}{\partial g}= \\
& -\left(\frac{1 / g}{g y+\overline{\mathcal{I}}_{\mathrm{p}} p_{\mathrm{p}}}+\frac{\overline{\mathcal{I}}_{\mathrm{p}} p_{\mathrm{p}} y}{\left(g y+\overline{\mathcal{I}}_{\mathrm{p}} p_{\mathrm{p}}\right)^{2}}\right) \exp \left(-\frac{1}{g y}\right)<0,
\end{aligned}
$$

thus $P_{\text {out }, \mathrm{s}}$ is monotonically decreasing with respect to $g$. Since $P_{\text {out }, \mathrm{s}}(g, y)$ is symmetrical with respect to $g$ and $y$, then it is also monotonically decreasing with respect to $y$. To prove $P_{\text {out }, \mathrm{s}}(g, y)$ is monotonically decreasing in $\mathcal{C}_{x}$, we need to prove that $g\left(\mathcal{C}_{x}\right)$ and $y\left(\mathcal{C}_{x}\right)$ are monotonically increasing with respect to $\mathcal{C}_{x}$.

To study the behavior of $g\left(\mathcal{C}_{x}\right)$, we find $\frac{\partial g\left(\mathcal{C}_{x}\right)}{\partial \mathcal{C}_{x}}$, which can be written as

$$
\begin{gathered}
\frac{\partial g\left(\mathcal{C}_{x}\right)}{\partial \mathcal{C}_{x}}=-\frac{\bar{\gamma}_{\mathrm{s}} \mathcal{C}_{x}}{\overline{\mathcal{I}}_{\mathrm{s}} \Psi_{\mathrm{p}}\left(\mathcal{C}_{x}\right) \sqrt{1-\mathcal{C}_{x}^{2}}}\left(\frac{\exp \left(-\Psi_{\mathrm{p}}(0)\right)}{1-P_{\text {out }, \text { th }}}-1\right) \\
-\frac{\partial \Psi_{\mathrm{p}}\left(\mathcal{C}_{x}\right)}{\partial \mathcal{C}_{x}} \frac{\bar{\gamma}_{\mathrm{s}} \sqrt{1-\mathcal{C}_{x}^{2}}}{\overline{\mathcal{I}}_{\mathrm{s}} \Psi_{\mathrm{p}}^{2}\left(\mathcal{C}_{x}\right)}\left(\frac{\exp \left(-\Psi_{\mathrm{p}}(0)\right)}{1-P_{\text {out th }}}-1\right) .
\end{gathered}
$$


By evaluating $\frac{\partial \Psi_{\mathrm{p}}\left(\mathcal{C}_{x}\right)}{\partial \mathcal{C}_{x}}$, (66) can be simplified to be

$$
\begin{aligned}
\frac{\partial g\left(\mathcal{C}_{x}\right)}{\partial \mathcal{C}_{x}}= & \frac{\bar{\gamma}_{\mathrm{s}} \mathcal{C}_{x}}{\overline{\mathcal{I}}_{\mathrm{s}} \Psi_{\mathrm{p}}\left(\mathcal{C}_{x}\right) \sqrt{1-\mathcal{C}_{x}^{2}}}\left(\frac{\exp \left(-\Psi_{\mathrm{p}}(0)\right)}{1-P_{\text {out }, \text { th }}}-1\right) \\
& \times\left(\frac{\Gamma_{\mathrm{p}}\left(1-\mathcal{C}_{x}^{2}\right)}{\Psi_{\mathrm{p}}\left(\mathcal{C}_{x}\right) p_{\mathrm{p}} \bar{\gamma}_{\mathrm{p}} \sqrt{1+\Gamma_{\mathrm{p}}\left[1-\mathcal{C}_{x}^{2}\right]}}-1\right) .
\end{aligned}
$$

To judge on the behavior of $g\left(\mathcal{C}_{x}\right)$, we substitute $\Psi_{\mathrm{p}}\left(\mathcal{C}_{x}\right)$ in (67) obtaining

$$
\begin{gathered}
\frac{\partial g\left(\mathcal{C}_{x}\right)}{\partial \mathcal{C}_{x}}=\frac{\bar{\gamma}_{\mathrm{s}} \mathcal{C}_{x}}{\overline{\mathcal{I}}_{\mathrm{s}} \Psi_{\mathrm{p}}\left(\mathcal{C}_{x}\right)} \sqrt{\sqrt{1-\mathcal{C}_{x}^{2}}}\left(\frac{\exp \left(-\Psi_{\mathrm{p}}(0)\right)}{1-P_{\text {out }, \text { th }}}-1\right) \\
\times \frac{1}{\sqrt{1+\Gamma_{\mathrm{p}}\left[1-\mathcal{C}_{x}^{2}\right]}}
\end{gathered}
$$

Since $\Psi_{\mathrm{p}}\left(\mathcal{C}_{x}\right)>0$ and $\exp \left(-\Psi_{\mathrm{p}}(0)\right)>\left(1-P_{\text {out }, \text { th }}\right)$, then $g\left(\mathcal{C}_{x}\right)$ is monotonically increasing with respect to $\mathcal{C}_{x}$. As for $y\left(\mathcal{C}_{x}\right)$, the $\frac{\partial y\left(\mathcal{C}_{x}\right)}{\partial \mathcal{C}_{x}}$ is found to be

$$
\begin{aligned}
& \frac{\partial y\left(\mathcal{C}_{x}\right)}{\partial \mathcal{C}_{x}}=\frac{\mathcal{C}_{x}}{\left(\sqrt{1+\Gamma_{\mathrm{s}}\left(1-\mathcal{C}_{x}^{2}\right)}-1\right) \sqrt{1-\mathcal{C}_{x}^{2}}} \\
& \quad \times\left(2 \sqrt{1+\Gamma_{\mathrm{s}}\left(1-\mathcal{C}_{x}^{2}\right)}+1\right)>0,
\end{aligned}
$$

which proves that $y\left(\mathcal{C}_{x}\right)$ is a monotonically increasing function. Therefore, from (65)-(69), $P_{\text {out, s }}$ is proved to be monotonically decreasing with respect to $\mathcal{C}_{x}$.

\section{REFERENCES}

[1] Q. Zhao and B. M. Sadler, "A survey of dynamic spectrum access," IEEE Signal Process. Mag., vol. 24, no. 3, pp. 79-89, May 2007.

[2] V. R. Cadambe, S. A. Jafar, and C. Wang, "Interference alignment with asymmetric complex signalingsettling the høst-madsen-nosratinia conjecture," IEEE Trans. Inf. Theory, vol. 56, no. 9, pp. 4552-4565, Sep. 2010

[3] Y. Zeng, C. M. Yetis, E. Gunawan, Y. L. Guan, and R. Zhang, "Transmit optimization with improper Gaussian signaling for interference channels," IEEE Trans. Signal Process., vol. 61, no. 11, pp. 2899-2913, Jun. 2013.

[4] F. D. Neeser and J. L. Massey, "Proper complex random processes with applications to information theory," IEEE Trans. Inf. Theory, vol. 39, no. 4, pp. 1293-1302, Jul. 1993.

[5] B. Picinbono, "On circularity," IEEE Trans. Signal Process., vol. 42, no. 12, pp. 3473-3482, Dec. 1994.

[6] - "Second-order complex random vectors and normal distributions," IEEE Trans. Signal Process., vol. 44, no. 10, pp. 2637-2640, Oct. 1996.

[7] B. Picinbono and P. Bondon, "Second-order statistics of complex signals," IEEE Trans. Signal Process., vol. 45, no. 2, pp. 411-420, Feb. 1997.

[8] P. J. Schreier and L. L. Scharf, "Second-order analysis of improper complex random vectors and processes," IEEE Trans. Signal Process., vol. 51, no. 3, pp. 714-725, Mar. 2003.

[9] T. Adali, P. J. Schreier, and L. L. Scharf, "Complex-valued signal processing: The proper way to deal with impropriety," IEEE Trans. Signal Process., vol. 59, no. 11, pp. 5101-5125, Nov. 2011.

[10] P. J. Schreier, L. L. Scharf, and A. Hanssen, "A generalized likelihood ratio test for impropriety of complex signals," IEEE Signal Process. Lett., vol. 13, no. 7, pp. 433-436, Jul. 2006.

[11] A. T. Walden and P. Rubin-Delanchy, "On testing for impropriety of complex-valued Gaussian vectors," IEEE Trans. Signal Process., vol. 57, no. 3, pp. 825-834, Mar. 2009.

[12] P. J. Schreier, "Bounds on the degree of impropriety of complex random vectors," IEEE Signal Process. Lett., vol. 15, pp. 190-193, 2008.

[13] G. Tauböck, "Complex-valued random vectors and channels: entropy, divergence, and capacity," IEEE Trans. Inf. Theory, vol. 58, no. 5, pp. 2729-2744, May 2012.

[14] Z. Ho and E. Jorswieck, "Improper Gaussian signaling on the two-user SISO interference channel," IEEE Trans. Wireless Commun., vol. 11, no. 9, pp. 3194-3203, Sep. 2012.
[15] Y. Zeng, R. Zhang, E. Gunawan, and Y. Guan, "Optimized transmission with improper Gaussian signaling in the K-user MISO interference channel," IEEE Trans. Wireless Commun., vol. 12, no. 12, pp. 63036313, Dec. 2013.

[16] E. Kurniawan and S. Sun, "Improper gaussian signaling scheme for the Z-interference channel," IEEE Trans. Wireless Commun., vol. 14, no. 7, pp. 3912-3923, Jul. 2015.

[17] S. Lagen, A. Agustin, and J. Vidal, "Improper Gaussian signaling for the Z-interferece channel," in in Proc. IEEE International Conference on Acoustics, Speech and Signal Processing (ICASSP), Florence, Italy, May 2014, pp. 1140-1144.

[18] C. Huang and S. A. Jafar, "Degrees of freedom of the MIMO interference channel with cooperation and cognition," IEEE Trans. Inf. Theory, vol. 55, no. 9, pp. 4211-4220, Sep. 2009.

[19] N. Devroye, P. Mitran, and V. Tarokh, "Achievable rates in cognitive radio channels," IEEE Trans. Inf. Theory, vol. 52, no. 5, pp. 1813-1827, May 2006.

[20] C. Lameiro, I. Santamaría, and P. J. Schreier, "Benefits of improper signaling for underlay cognitive radio," IEEE Wireless Commun. Lett., vol. 4, no. 1, pp. 22-25, Feb. 2015.

[21] M. Gaafar, O. Amin, W. Abediseid, and M.-S. Alouini, "Spectrum sharing opportunities of full-duplex systems using improper Gaussian signaling," in in Proc. IEEE International Symposium on Personal, Indoor and Mobile Radio Communications (PIMRC), Hong Kong, Sep. 2015.

[22] O. Amin, W. Abediseid, and M.-S. Alouini, "Outage performance of cognitive radio systems with improper Gaussian signaling," in Proc. IEEE International Symposium on Information Theory (ISIT), Hong Kong, Jun. 2015.

[23] P. J. Schreier and L. L. Scharf, Statistical signal processing of complexvalued data: the theory of improper and noncircular signals. Cambridge University Press, 2010.

[24] A. Goldsmith, Wireless communications. Cambridge university press, 2005.

[25] K. Cumanan, R. Zhang, and S. Lambotharan, "A new design paradigm for MIMO cognitive radio with primary user rate constraint," IEEE Commun. Lett., vol. 16, no. 5, pp. 706-709, May 2012.

[26] H. Holma and A. Toskala, WCDMA for UMTS: HSPA evolution and LTE. John Wiley \& Sons, 2010.

[27] L. Musavian and S. Aïssa, "Capacity of spectrum-sharing channels with minimum-rate requirements," in IEEE International Conference on Communications (ICC), Beijing, China, May 2008, pp. 4639-4643.

[28] R. M. Corless, G. H. Gonnet, D. E. Hare, D. J. Jeffrey, and D. E. Knuth, "On the lambertw function," Advances in Computational Mathematics, vol. 5, no. 1, pp. 329-359, Dec. 1996.

[29] A. A. Puntambekar, Analysis and Design of Algorithms. Technical Publications, 2008.

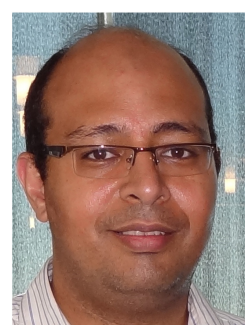

Osama Amin (S'07, M'11, SM'15) received B. Sc. degree in Electrical and Electronics Engineering from Aswan University, Aswan, Egypt, in 2000, M. Sc. degree in Electrical Engineering from Assiut University, Assiut, Egypt in 2004 and Ph.D. degree in Electrical and Computer Engineering, University of Waterloo, Canada in 2010. In June 2012, he joined Assiut University as an Assistant Professor in the Electrical and Electronics Engineering department. Currently, he is with King Abdullah University of Science and Technology (KAUST), Thuwal, Makkah, Kingdom of Saudi Arabia. His general research interests lie in communications systems and signal processing for communications with special emphasis on wireless applications. Specific research areas include green communications, cognitive radio, cooperative communications, and channel estimation.

Dr. Amin is an Editor for the IEEE Communications Letters. He has served as a technical program committee (TPC) member for IEEE VTC, CROWNCOM, PIMRC, and ISSPIT conferences. He has served also as coorganizer and a co-chair of the Next Generation Green ICT and 5G Networking (GreeNets) 2015 in the IEEE International Conference on Ubiquitous Wireless Broadband (ICUWB) in Montreal, Canada. 


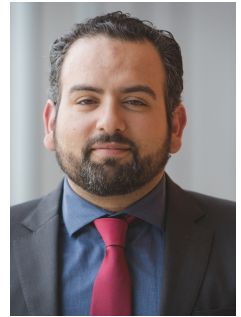

Walid Abediseid (S'04, M'11) was born in Etobicoke, Ontario, Canada. He received the B. Sc. and M. Sc. degrees in Electrical Engineering from the University of Ottawa, Canada, in 2004 and 2007, respectively. He then received his Ph.D. from the Department of Electrical and Computer Engineering, University of Waterloo in 2011. He was a postdoctoral fellow at King Abdullah University of Science and Technology (KAUST), Thuwal, Makkah Province, Saudi Arabia, from December 2011 to December 2013. His research interests included coding and information theory, MIMO wireless communication systems, lattice applications for digital and wireless communications, detection and estimation.

In January 2014, Dr. Abediseid joined the Saudi Initiatives department at KAUST where he holds the academic development specialist of the Saudi Research Science Institute. He also serves as a tutor during the SRSI summer program that provides support to students conducting research in STEM fields.

Dr. Abediseid was a recipient of many prestigious awards during his graduate studies such as the Research In Motion Graduate Scholarship from 2008 to 2009, and the NSERC Alexander Graham Bell Canada Graduate Scholarshipone of Canada's most prestigious graduate awards from 2009 to 2011.

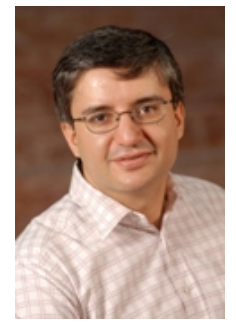

Mohamed-Slim Alouini (S'94, M'98, SM'03, F'09) was born in Tunis, Tunisia. He received the Ph.D. degree in Electrical Engineering from the California Institute of Technology (Caltech), Pasadena, CA, USA, in 1998. He served as a faculty member in the University of Minnesota, Minneapolis, MN, USA, then in the Texas A\&M University at Qatar, Education City, Doha, Qatar before joining King Abdullah University of Science and Technology (KAUST), Thuwal, Makkah Province, Saudi Arabia as a Professor of Electrical Engineering in 2009. His current research interests include the modeling, design, and performance analysis of wireless communication systems. 